\title{
IMPROVED PARAMETRIX IN THE GLANCING REGION FOR THE INTERIOR DIRICHLET-TO-NEUMANN MAP
}

\author{
GEORGI VODEV
}

\begin{abstract}
We study the semi-classical microlocal structure of the Dirichlet-to-Neumann map for an arbitrary compact Riemannian manifold with a non-empty smooth boundary. We build a new, improved parametrix in the glancing region compaired with that one built in [9], [12. We also study the way in which the parametrix depends on the refraction index. As a consequence, we improve the transmission eigenvalue-free regions obtained in [12] in the isotropic case when the restrictions of the refraction indices on the boundary coincide.
\end{abstract}

\section{Introduction AND STATEMENT OF RESUlts}

Let $(X, \mathcal{G})$ be a compact Riemannian manifold of dimension $d=\operatorname{dim} X \geq 2$ with a nonempty smooth boundary $\partial X$ and let $\Delta_{X}$ denote the negative Laplace-Beltrami operator on $(X, \mathcal{G})$. Given a function $f \in H^{m+1}(\partial X)$, let $u$ solve the equation

$$
\begin{cases}\left(\Delta_{X}+\lambda^{2} n(x)\right) u=0 & \text { in } X, \\ u=f & \text { on } \partial X,\end{cases}
$$

where $\lambda \in \mathbf{C}, 1 \ll|\operatorname{Im} \lambda| \ll \operatorname{Re} \lambda$ and $n \in C^{\infty}(\bar{X})$ is a strictly positive function called refraction index. The Dirichlet-to-Neumann (DN) map

$$
\mathcal{N}(\lambda ; n): H^{m+1}(\partial X) \rightarrow H^{m}(\partial X)
$$

is defined by

$$
\mathcal{N}(\lambda ; n) f:=\left.\partial_{\nu} u\right|_{\partial X}
$$

where $\nu$ is the unit inner normal to $\partial X$. Introduce the semi-classical parameter $0<h \ll 1$ such that $\operatorname{Re}(h \lambda)^{2}=1$ and set $z=(h \lambda)^{2}=1+i \operatorname{Im} z$ with $0<|\operatorname{Im} z| \leq 1$. Then the problem (1.1) can be rewritten as follows

$$
\begin{cases}\left(h^{2} \Delta_{X}+z n(x)\right) u=0 & \text { in } \quad X, \\ u=f & \text { on } \partial X .\end{cases}
$$

Define the semi-classical DN map, $N(h, z)$, by

$$
N(h, z) f:=\left.\mathcal{D}_{\nu} u\right|_{\partial X}=-i h \mathcal{N}(\lambda ; n) f
$$

where $\mathcal{D}_{\nu}:=-i h \partial_{\nu}$. Denote by $\Delta_{\partial X}$ the negative Laplace-Beltrami operator on $\left(\partial X, \mathcal{G}_{0}\right)$, which is a Riemannian manifold without boundary of dimension $d-1$, where $\mathcal{G}_{0}$ is the Riemannian metric on $\partial X$ induced by the metric $\mathcal{G}$. Let $r_{0}\left(x^{\prime}, \xi^{\prime}\right) \geq 0$ be the principal symbol of $-\Delta_{\partial X}$ written in the coordinates $\left(x^{\prime}, \xi^{\prime}\right) \in T^{*} \partial X$. The glancing region, $\Sigma$, for the problem (1.2) (resp. (1.1)) is defined by

$$
\Sigma:=\left\{\left(x^{\prime}, \xi^{\prime}\right) \in T^{*} \partial X: r_{0}\left(x^{\prime}, \xi^{\prime}\right)=n_{0}\left(x^{\prime}\right)\right\}, \quad n_{0}:=\left.n\right|_{\partial X} .
$$

Our goal in the present paper is to build a semi-classical parametrix for the operator $N(h, z)$ in a neighbourhood of $\Sigma$ for $|\operatorname{Im} z| \geq h^{2 / 3-\epsilon}, 0<\epsilon \ll 1$ being arbitrary. Since $h \sim|\lambda|^{-1}$, it is easy to see that on the $\lambda$ - plane this region takes the form $|\operatorname{Im} \lambda| \geq|\lambda|^{1 / 3+\epsilon},|\lambda| \gg 1$. Note 
that such a parametrix has been previously constructed in [9], 12] for $|\operatorname{Im} z| \geq h^{1 / 2-\epsilon}$ (this corresponds to the region $|\operatorname{Im} \lambda| \geq|\lambda|^{1 / 2+\epsilon},|\lambda| \gg 1$, on the $\lambda$ - plane). Roughly speaking, the smaller $|\operatorname{Im} z|$ is, the harder is to construct a parametrix for $N(h, z)$. Note also that a semiclassical parametrix for the operator $N(h, z)$ in a neighbourhood of $\Sigma$ has been built in [10] for $|\operatorname{Im} z| \geq h^{1-\epsilon}$ (which corresponds to the region $|\operatorname{Im} \lambda| \geq|\lambda|^{\epsilon},|\lambda| \gg 1$, on the $\lambda$ - plane) but under the additional assumption that the boundary $\partial X$ is strictly concave. Under this condition another semi-classical parametrix was built in [5] for $|\operatorname{Im} z| \sim h^{2 / 3}$.

It has been shown in [9] that $N(h, z) \in O P S_{\delta, \delta}^{0,1}(\partial X)$ (see Section 2 for the definition of the $h-\Psi$ DOs of class $\left.O P S_{\delta_{1}, \delta_{2}}^{k_{1}, k_{2}}\right)$ for $|\operatorname{Im} z| \geq h^{1 / 2-\epsilon}$ with $\delta=1 / 2-\epsilon$, and a principal symbol, $\rho$, defined by

$$
\rho\left(x^{\prime}, \xi^{\prime} ; z\right)=\sqrt{-r_{0}\left(x^{\prime}, \xi^{\prime}\right)+z n_{0}\left(x^{\prime}\right)}, \quad \operatorname{Im} \rho>0 .
$$

Moreover, outside the glancing region the operator $N(h, z)$ belongs to a much better class, due to the fact that there $|\rho|$ is lower bounded by a positive constant. To be more precise, we choose a cut-off function $\chi \in C_{0}^{\infty}\left(T^{*} \partial X\right)$, independent of $h$ and $z$, such that $\chi=1$ in a small, $h-$ independent neighbourhood of $\Sigma$. It follows from the analysis in [9] that $N(h, z) \operatorname{Op}_{h}(1-\chi) \in$ $O P S_{0,0}^{0,1}(\partial X)$ for $|\operatorname{Im} z| \geq h^{1-\epsilon}$ with a principal symbol $\rho(1-\chi)$. In other words, the condition $|\operatorname{Im} z| \geq h^{1 / 2-\epsilon}$ is only required in 9 ] to study the operator $N(h, z)$ near $\Sigma$. Note that the full symbol of $N(h, z)$ depends on the functions $n_{k}=\left.\partial_{\nu}^{k} n\right|_{\partial X}, k=0,1, \ldots$, and their derivatives. The way in which the parametrix of the operator $N(h, z)$ depends on $n_{k}$ is studied in [12]. Near the glancing region the analysis in [12] again requires the condition $|\operatorname{Im} z| \geq h^{1 / 2-\epsilon}$, while outside $\Sigma$ it works for $|\operatorname{Im} z| \geq h^{1-\epsilon}$.

In the present paper we will extend the analysis near $\Sigma$ to the larger region $|\operatorname{Im} z| \geq h^{2 / 3-\epsilon}$. The first difficulty to deal with is to give a reasonable definition of the operator $\operatorname{Op}_{h}(\rho)$ when $|\operatorname{Im} z| \sim h^{2 / 3-\epsilon}$. Indeed, in this case $\chi \rho \in S_{\delta, \delta}^{0,0}(\partial X)$ with $\delta=2 / 3-\epsilon>1 / 2$ and we do not have a good calculus for $h-\Psi D O$ s with symbols in this class. To overcome this difficulty we use the second microlocalization with respect to $\Sigma$. Note that this approach proved very usefull when studying the resonances near cubic curves in the case of scattering by strictly convex obstacles (see [6]). It has been also successfully used in [3] to study the location of the resonances for various exterior transmission problems associated to transparent strictly convex obstacles. It has already been used in the contex of the interior DN map in [5] and [10] to build a parametrix when the boundary $\partial X$ is strictly concave. Here we will use it for an arbitrary Riemannian manifold. Roughly speaking, it consists of using $h$-FIOs to transform our problem (1.2) microlocally near the boundary into a simpler equation (see the model equation (2.3) in Section 2) for which it is easier to construct a microlocal parametrix. Then the global parametrix is obtained by using a suitable partition of the unity on $\Sigma$. More precisely, let $\mathcal{W} \subset T^{*} \partial X$ be a small neighbourhood of $\Sigma$ such that $\operatorname{supp} \chi \subset \mathcal{W}$ and cover $\mathcal{W}$ with a finite number of sufficiently small, open domains, $\mathcal{W}_{j} \subset T^{*} \partial X, j=1, \ldots, J, \Sigma \subset \mathcal{W} \subset \cup_{j=1}^{J} \mathcal{W}_{j}$. Choose functions $\chi_{j}, \psi_{j} \in C_{0}^{\infty}\left(\mathcal{W}_{j}\right)$ such that $\psi_{j}=1$ on $\operatorname{supp} \chi_{j}$ and $\chi=\sum_{j=1}^{J} \chi_{j}$. It is well-known (e.g. see Theorem 12.3 of [13]) that there are an open, bounded domain $Y_{j} \subset \mathbf{R}^{d-1}$ and a symplectomorphism $\kappa_{j}: \mathcal{W}_{j} \rightarrow T^{*} Y_{j}$ such that in the new coordinates $(y, \eta)=\kappa_{j}\left(x^{\prime}, \xi^{\prime}\right)$ we have

$$
\eta_{1}=n_{0}^{-1} r_{0}\left(x^{\prime}, \xi^{\prime}\right)-1
$$

and $\kappa_{j}\left(\Sigma \cap \mathcal{W}_{j}\right)=\left\{\eta_{1}=0\right\}$. We can write the function $\rho$ as follows

$$
\rho=n_{0}^{1 / 2} \varrho \circ \kappa_{j}^{-1}
$$


where

$$
\varrho\left(\eta_{1}\right)=\sqrt{-\eta_{1}+i \operatorname{Im} z}, \quad \operatorname{Im} \varrho>0 .
$$

Let $U_{j}: L^{2}\left(\pi \mathcal{W}_{j}\right) \rightarrow L^{2}\left(Y_{j}\right)$ be an elliptic, zero-order $h$-FIO associated to $\kappa_{j}$, where $\pi$ : $T^{*} \partial X \rightarrow \partial X$ denotes the projection $\pi\left(x^{\prime}, \xi^{\prime}\right)=x^{\prime}$. Then we define the operators

$$
\widetilde{\mathrm{Op}}_{h}(\rho \chi)=\sum_{j=1}^{J} \mathrm{Op}_{h}\left(n_{0}^{1 / 2} \chi_{j}\right) U_{j}^{-1} \mathrm{Op}_{h}\left(\phi_{j} \varrho\right) U_{j} \mathrm{Op}_{h}\left(\psi_{j}\right): L^{2}(\partial X) \rightarrow L^{2}(\partial X)
$$

where $\phi_{j}\left(\eta_{1}\right) \in C_{0}^{\infty}$ is such that $\phi_{j}=1$ on $\operatorname{supp} \chi_{j} \circ \kappa_{j}$, and

$$
\widetilde{\mathrm{Op}}_{h}(\rho)=\mathrm{Op}_{h}(\rho(1-\chi))+\widetilde{\mathrm{Op}}_{h}(\rho \chi) .
$$

We can define similarly the operators $\widetilde{\mathrm{Op}}_{h}\left(\rho^{k} \chi\right)$ and $\widetilde{\mathrm{Op}}_{h}\left(\rho^{k}\right), k$ being an arbitrary integer. When $|\operatorname{Im} z| \geq h^{1 / 2-\epsilon}$ one can see that the operator $\widetilde{\mathrm{Op}}_{h}(\rho \chi)$ coincides with the standard $h-\Psi \mathrm{DO}$ $\mathrm{Op}_{h}(\rho \chi) \bmod \mathcal{O}\left(h^{\epsilon}\right)$. We refer to Section 4 of [6], Section 4 of [3] as well as Sections 10, 11 and 12 of [13] for more information about the properties of these operators. In the present paper we only make use of some very basic properties of the $h-\Psi$ DOs and $h-$ FIOs.

Thus we reduce the parametrix construction in the glancing region to building a parametrix for a model equation (see Section 3). On the other hand, the parametrix construction for the model equation is carried out in Section 2 following that one in Section 6 of [10] in the case of strictly concave boundary. Note that the model equation in [10] is much simpler than this one we study in the present paper. This is due to the fact that the strict concavity condition allows to use the symplectic normal form proved in [7]. No such normal forms exist, however, in the general case and therefore we have to work with a model equation which is relatively complicated. Nevertheless, we show that the parametrix construction still works. As a consequence we get the following

Theorem 1.1. Let $|\operatorname{Im} z| \geq h^{2 / 3-\epsilon}, 0<\epsilon \ll 1$. Then we have

$$
\left\|N(h, z) \mathrm{Op}_{h}(\chi)-\widetilde{\mathrm{Op}}_{h}(\rho \chi)\right\| \lesssim h|\operatorname{Im} z|^{-1} .
$$

Hereafter $\|\cdot\|$ denotes the $L^{2}(\partial X) \rightarrow L^{2}(\partial X)$ norm. This theorem is a significant improvement upon the results in [9] and [12]. Indeed, an analog of this theorem has been proved in [9] and [12] but for $|\operatorname{Im} z| \geq h^{1 / 2-\epsilon}$ and with an worse bound in the right-hand side of (1.3) (with $|\operatorname{Im} z|^{-3 / 2}$ in place of $\left.|\operatorname{Im} z|^{-1}\right)$. In fact, we get a full expansion in powers of $h$ of the operator $N \mathrm{Op}_{h}(\chi)$ and we study the way in which it depends on the functions $n_{k}$ (see Theorem 3.1).

Outside the glancing region we have a better bound for the DN map. Indeed, it has been proved in [9] and [12] that for $|\operatorname{Im} z| \geq h^{1-\epsilon}$ the following estimate holds:

$$
\left\|N(h, z) \mathrm{Op}_{h}(1-\chi)-\mathrm{Op}_{h}(\rho(1-\chi))\right\| \lesssim h .
$$

Combining (1.3) and (1.4) we get the following

Theorem 1.2. Let $|\operatorname{Im} z| \geq h^{2 / 3-\epsilon}, 0<\epsilon \ll 1$. Then we have

$$
\left\|N(h, z)-\widetilde{\mathrm{Op}}_{h}(\rho)\right\| \lesssim h|\operatorname{Im} z|^{-1} .
$$

This theorem provides a good approximation of the DN map by an $h-\Psi D O$ as long as $|\operatorname{Im} z| \geq h^{2 / 3-\epsilon}$. Even better approximations are given in Theorem 3.5. For many applications, however, one needs to have some less accurate approximation of the DN map but for smaller $|\operatorname{Im} z|$. Indeed, such an approximation has been proved in [11] for $|\operatorname{Im} z| \geq C h$, provided the constant $C>0$ is taken big enough. Having high-frequency approximations of the DN map proves very usefull when studying the location of the complex eigenvalues associated to boundary 
value problems with dissipative boundary conditions or to interior transmission problems. In particular, this proves crucial to get parabolic transmission eigenvalue-free regions (see [9], [10], [11], [12]). As an application of our parametrix we improve the transmission eigenvalue-free region obtained in [12] in the case of the degenerate isotropic interior transmission problem (see Theorem 4.1).

\section{Parametrix Construction for the model equation}

Let $Y \subset \mathbf{R}^{d-1}, d \geq 2$, be a bounded, open domain. Given $k_{1}, k_{2} \in \mathbf{R}, \delta_{1}, \delta_{2} \geq 0$, define the class of symbols $S_{\delta_{1}, \delta_{2}}^{k_{1}, k_{2}}(Y)$ as being the set of all functions $a \in C^{\infty}\left(T^{*} Y\right)$, $\operatorname{supp}_{y} a(y, \eta) \subset Y$, depending on a semi-classical parameter $0<h \ll 1$ and satisfying the bounds

$$
\left|\partial_{y}^{\alpha} \partial_{\eta}^{\beta} a(y, \eta)\right| \leq C_{\alpha, \beta} h^{-k_{1}-\delta_{1}|\alpha|-\delta_{2}|\beta|}\langle\eta\rangle^{k_{2}-|\beta|}
$$

for all multi-indices $\alpha$ and $\beta$. We then define the $h-\Psi D O$ with a symbol $a$ by

$$
a\left(y, \mathcal{D}_{y}\right) f:=\left(\operatorname{Op}_{h}(a) f\right)(y):=(2 \pi h)^{-d+1} \int_{T^{*} Y} e^{-\frac{i}{h}\langle y-w, \eta\rangle} a(y, \eta) f(w) d w d \eta
$$

where $\mathcal{D}_{y}:=-i h \partial_{y}$. We will denote by $O P S_{\delta_{1}, \delta_{2}}^{k_{1}, k_{2}}(Y)$ the set of all operators $\operatorname{Op}_{h}(a)$ with symbols $a \in S_{\delta_{1}, \delta_{2}}^{k_{1}, k_{2}}(Y)$. Since $Y$ is bounded, we have the following simple criteria for an $h-\Psi \mathrm{DO}$ to be bounded on $L^{2}(Y)$ :

$$
\left\|\mathrm{Op}_{h}(a)\right\|_{L^{2}(Y) \rightarrow L^{2}(Y)} \leq C \sum_{0 \leq|\alpha| \leq d} \sup _{y, \eta}\left|\partial_{y}^{\alpha} a(y, \eta)\right|
$$

where $C>0$ is a constant independent of $a$ and $h$. In particular, (2.1) implies that if $a \in$ $S_{0, \delta_{2}}^{k_{1}, 0}(Y)$, then $\mathrm{Op}_{h}(a)=\mathcal{O}\left(h^{-k_{1}}\right): L^{2}(Y) \rightarrow L^{2}(Y)$. Note that (2.1) is no longer true if $Y=$ $\mathbf{R}^{d-1}$. Indeed, in that case one also needs some information concerning the derivatives with respect to the variable $\eta$.

Given two symbols $a$ and $b$ and an integer $M \geq 1$, set

$$
E_{M}(a, b)=\sum_{0 \leq|\alpha| \leq M} \frac{(-i h)^{|\alpha|}}{|\alpha| !} \partial_{\eta}^{\alpha} a(y, \eta) \partial_{y}^{\alpha} b(y, \eta) .
$$

It is easy to see that if $a \in S_{\delta_{1}, \delta_{2}}^{k_{1}, k_{2}}(Y), b \in S_{\delta_{1}^{\prime}, \delta_{2}^{\prime}}^{k_{1}^{\prime}, k_{2}^{\prime}}(Y)$ with $\delta_{2}+\delta_{1}^{\prime} \leq 1$, then $E_{M}(a, b) \in S_{\delta_{1}^{\sharp}, \delta_{2}^{\sharp}}^{k_{2}^{\sharp}, k_{2}^{\sharp}}(Y)$, where $k_{j}^{\sharp}=k_{j}+k_{j}^{\prime}, \delta_{j}^{\sharp}=\max \left\{\delta_{j}, \delta_{j}^{\prime}\right\}, j=1,2$. The following proposition follows from the calculus developed in Section 7 of [2].

Proposition 2.1. Let $a \in S_{\delta_{1}, \delta_{2}}^{k_{1}, 0}(Y)$ and let $b \in C^{\infty}\left(T^{*} Y\right)$ satisfy

$$
\left|\partial_{y}^{\alpha} b(y, \eta)\right| \leq C_{\alpha} h^{-k_{2}-\delta_{1}^{\prime}|\alpha|}
$$

for all multi-indices $\alpha$. If $\delta_{2}+\delta_{1}^{\prime}<1$, then there is $M_{0}>0$ such that for all $M \geq M_{0}$ we have the bound

$$
\left\|\mathrm{Op}_{h}(a) \mathrm{Op}_{h}(b)-\mathrm{Op}_{h}\left(E_{M}(a, b)\right)\right\|_{L^{2}(Y) \rightarrow L^{2}(Y)} \lesssim h^{M\left(1-\delta_{2}-\delta_{1}^{\prime}\right) / 2} .
$$

Indeed, (2.2) is an easy consequence of the inequality (7.17) of [2] and the bound (2.1) (e.g. see Section 4 of [10]). 
Let $y=\left(y_{1}, \ldots, y_{d-1}\right)$ be coordinates in $Y$ and let $\eta=\left(\eta_{1}, \ldots, \eta_{d-1}\right)$ be the dual variables. Let $\mu \in \mathbf{R}$ be a parameter satisfying $h^{2 / 3-\epsilon} \leq|\mu| \leq 1,0<\epsilon \ll 1$ being arbitrary. Consider in $(0,1) \times Y$ the operator

$$
P_{0}(h, \mu)=\mathcal{D}_{t}^{2}+\mathcal{D}_{y_{1}}-i \mu+m\left(t, y, \mathcal{D}_{y} ; h, \mu\right)
$$

where $t \in(0,1), \mathcal{D}_{t}:=-i h \partial_{t}, \mathcal{D}_{y_{1}}:=-i h \partial_{y_{1}}$. We suppose that there are functions $m_{j} \in$ $C^{\infty}\left([0,1] \times T^{*} Y\right), j=0,1, \ldots$, independent of $h$, such that for every integer $\nu \geq 0$ the function $m$ can be written in the form

$$
m(t, y, \eta ; h, \mu)=\sum_{j=0}^{\nu} h^{j} m_{j}(t, y, \eta ; \mu)+h^{\nu+1} m_{\nu+1}^{\sharp}(t, y, \eta ; h, \mu)
$$

where $m_{\nu+1}^{\sharp} \in S_{0,0}^{0,0}(Y)$ uniformly in $t, h$ and $\mu$. We also suppose that $\operatorname{Im} \partial_{t}^{\alpha} \partial_{y}^{\beta} m_{0}=\mathcal{O}(|\mu|)$ for all multi-indices $\alpha$ and $\beta$, and that for all integers $k, j \geq 0, \partial_{t}^{k} m_{j} \in S_{0,0}^{0,0}(Y)$ uniformly in $t$ and $\mu$. The Taylor expansion at $t=0$ gives, for every integer $\nu \geq 0$,

$$
m_{j}(t, y, \eta ; \mu)=\sum_{k=0}^{\nu} t^{k} m_{k, j}(y, \eta ; \mu)+t^{\nu+1} \widetilde{m}_{\nu+1, j}(t, y, \eta ; \mu)
$$

where $\widetilde{m}_{\nu+1, j} \in S_{0,0}^{0,0}(Y)$ uniformly in $t$ and $\mu$. We will write

$$
m \sim \sum_{j=0}^{\infty} h^{j} m_{j} \sim \sum_{k=0}^{\infty} \sum_{j=0}^{\infty} t^{k} h^{j} m_{k, j} .
$$

Finally, we suppose that the function $m_{0,0}:=m_{0}(0, y, \eta ; \mu)$ is identically zero on $T^{*} Y$. In other words, $m=\mathcal{O}(t+h)$ as $t \rightarrow 0$.

Our goal in this section is to build a parametrix, $\widetilde{u}$, for the solution to the following boundary value problem:

$$
\begin{cases}P_{0}(h, \mu) u(t, y)=0 & \text { in } \quad(0,1) \times Y, \\ u(0, y)=\phi\left(\mathcal{D}_{y_{1}}\right) f \quad \text { on } \quad Y,\end{cases}
$$

where $f \in L^{2}(Y)$ and $\phi \in C_{0}^{\infty}(\mathbf{R})$ is independent of $h$ and $\mu$, such that $\phi(\sigma)=1$ for $|\sigma| \leq 1$, $\phi(\sigma)=0$ for $|\sigma| \geq 2$. We will also study the way in which the parametrix depends on the functions $m_{k, j}$. We will be looking for $\widetilde{u}$ in the form

$$
\widetilde{u}(t, y)=(2 \pi h)^{-d+1} \int_{T^{*} Y} e^{-\frac{i}{h}(\langle y-w, \eta\rangle-\varphi(t, y, \eta ; \mu))} \Phi_{\epsilon, \delta}\left(t, \eta_{1} ; \mu\right) a(t, y, \eta ; h, \mu) f(w) d w d \eta
$$

where $\Phi_{\epsilon, \delta}=\phi\left(t / h^{\epsilon}\right) \phi\left(t /|\varrho|^{2} \delta\right), 0<\delta \ll 1$ being a parameter independent of $h$ and $\mu$ to be fixed later on and the function $\varrho$ is defined by

$$
\varrho\left(\eta_{1} ; \mu\right)=\sqrt{-\eta_{1}+i \mu}, \quad \operatorname{Im} \varrho>0 .
$$

Clearly, we have $|\varrho|^{2} \geq|\mu|$. We also have $|\varrho| \leq$ Const as long as $\eta_{1} \in \operatorname{supp} \phi$.

The phase $\varphi$ is complex-valued, independent of $h$, to be determined later on such that $\left.\varphi\right|_{t=0} \equiv$ 0 . The amplitude $a$ is of the form

$$
a=\sum_{j=0}^{M} h^{j} a_{j}
$$


where $M$ is an arbitrary sufficiently large integer and the functions $a_{j}$ are independent of $h$ to be determined later on such that $\left.a_{0}\right|_{t=0}=\phi\left(\eta_{1}\right),\left.a_{j}\right|_{t=0} \equiv 0$ for all $j \geq 1$. Thus we have $\widetilde{u}(0, y)=\phi\left(\mathcal{D}_{y_{1}}\right) f$. Furthermore, writing formally

we get

$$
\widetilde{u}=\mathrm{Op}_{h}\left(e^{i \varphi / h} \Phi_{\epsilon, \delta} a\right) f
$$

$$
P_{0}(h, \mu) \widetilde{u}=\mathrm{Op}_{h}\left(e^{i \varphi / h} \Phi_{\epsilon, \delta} A_{M}\right) f+\mathrm{Op}_{h}\left(e^{i \varphi / h} A_{M}^{\sharp}\right) f+\mathcal{E}_{M} f
$$

where

$$
\begin{gathered}
A_{M}=\left(\left(\partial_{t} \varphi\right)^{2}+\partial_{y_{1}} \varphi-\varrho^{2}-i h \partial_{t}^{2} \varphi\right) a-2 i h \partial_{t} \varphi \partial_{t} a-i h \partial_{y_{1}} a-h^{2} \partial_{t}^{2} a \\
+e^{-i \varphi / h} E_{M}\left(m, e^{i \varphi / h} a\right), \\
A_{M}^{\sharp}=\left(-h^{2} \partial_{t}^{2} \Phi_{\epsilon, \delta}-2 i h \partial_{t} \Phi_{\epsilon, \delta} \partial_{t} \varphi\right) a-2 h^{2} \partial_{t} \Phi_{\epsilon, \delta} \partial_{t} a, \\
\mathcal{E}_{M}=\mathrm{Op}_{h}(m) \mathrm{Op}_{h}\left(e^{i \varphi / h} \Phi_{\epsilon, \delta} a\right)-\mathrm{Op}_{h}\left(E_{M}\left(m, e^{i \varphi / h} \Phi_{\epsilon, \delta} a\right)\right) .
\end{gathered}
$$

It is easy to see that $A_{M}^{\sharp}=0$ for $0 \leq t \leq \min \left\{h^{\epsilon}, \delta|\rho|^{2}\right\}$. We will now expand the function $A_{M}$ in powers of $h$. The most difficult is to expand the last term. To do so we need the following

Lemma 2.2. For every multi-index $\beta$ we have the identity

$$
\frac{(-i)^{|\beta|}}{|\beta| !} \partial_{y}^{\beta}\left(e^{i \varphi / h}\right)=e^{i \varphi / h} \sum_{k=0}^{|\beta|} h^{-k} G_{k}^{(\beta)}(\varphi)
$$

where the functions $G_{k}^{(\beta)}$ are independent of $h, G_{0}^{(0)}=1, G_{0}^{(\beta)}=0$ for $|\beta| \geq 1$. For $k \geq 1$, $|\beta| \geq 1$ they are of the form

$$
G_{k}^{(\beta)}(\varphi)=\sum_{\left|\gamma_{j}\right| \geq 1,\left|\gamma_{1}\right|+\ldots+\left|\gamma_{k}\right|=|\beta|} c_{\gamma_{1}, \ldots, \gamma_{k} ; k, \beta} \prod_{j=1}^{k} \partial_{y}^{\gamma_{j}} \varphi
$$

where the coefficients $c_{\gamma_{1}, \ldots, \gamma_{k} ; k, \beta}$ are constants. In particular, if $\beta=\left(\beta_{1}, \ldots, \beta_{d-1}\right)$, then

$$
G_{|\beta|}^{(\beta)}(\varphi)=\frac{1}{|\beta| !} \prod_{j=1}^{d-1}\left(\frac{\partial \varphi}{\partial y_{j}}\right)^{\beta_{j}} .
$$

This lemma can be easilly proved by induction in $|\beta|$ and therefore we omit the details. Using (2.5) we can write

$$
\begin{gathered}
\frac{(-i)^{|\alpha|}}{|\alpha| !} \partial_{y}^{\alpha}\left(e^{i \varphi / h} a\right)=\sum_{0 \leq|\beta| \leq|\alpha|} c_{\alpha, \beta} \frac{(-i)^{|\alpha-\beta|}}{|\alpha-\beta| !} \partial_{y}^{\alpha-\beta}\left(e^{i \varphi / h}\right) \partial_{y}^{\beta} a \\
=e^{i \varphi / h} \sum_{0 \leq|\beta| \leq|\alpha|} \sum_{k=0}^{|\alpha|-|\beta|} c_{\alpha, \beta} h^{-k} G_{k}^{(\alpha-\beta)}(\varphi) \partial_{y}^{\beta} a \\
=e^{i \varphi / h} h^{-|\alpha|} \sum_{\nu=0}^{|\alpha|} h^{\nu} \sum_{|\beta| \leq \nu} c_{\alpha, \beta} G_{|\alpha|-\nu}^{(\alpha-\beta)}(\varphi) \partial_{y}^{\beta} a
\end{gathered}
$$

where the coefficients $c_{\alpha, \beta}$ are constants. Thus we get the expansion

$$
e^{-i \varphi / h} \frac{(-i h)^{|\alpha|}}{|\alpha| !} \partial_{y}^{\alpha}\left(e^{i \varphi / h} a\right)=G_{|\alpha|}^{(\alpha)}(\varphi) a+\sum_{\nu=1}^{M+|\alpha|} h^{\nu} a_{\nu}^{(\alpha)}
$$


where

$$
a_{\nu}^{(\alpha)}=\sum_{\nu^{\prime}=(\nu-|\alpha|)_{+}}^{\nu-1} \sum_{|\beta| \leq \nu-\nu^{\prime}} c_{\alpha, \beta} G_{|\alpha|-\nu+\nu^{\prime}}^{(\alpha-\beta)}(\varphi) \partial_{y}^{\beta} a_{\nu^{\prime}}
$$

where $b_{+}:=\max \{0, b\}$. Clearly, we have $a_{\nu}^{(0)}=0$. We now expand the function $m$ as

$$
m=\sum_{j=0}^{M+1} h^{j} m_{j}+h^{M+2} m_{M+2}^{\sharp} .
$$

Using the above identities we can write

$$
e^{-i \varphi / h} E_{M}\left(m, e^{i \varphi / h} a\right)=g_{M}\left(m_{0}, \varphi\right) a+\sum_{j=0}^{M} h^{j+1} E_{j}^{(M)}+h^{M+2} \widetilde{E}_{M+2}
$$

where

$$
\begin{gathered}
g_{M}\left(m_{0}, \varphi\right)=\sum_{0 \leq|\alpha| \leq M} \partial_{\eta}^{\alpha} m_{0} G_{|\alpha|}^{(\alpha)}(\varphi), \\
E_{j}^{(M)}=\sum_{0 \leq|\alpha| \leq M} \sum_{\ell=0}^{j} \partial_{\eta}^{\alpha} m_{\ell} a_{j+1-\ell}^{(\alpha)}+\sum_{0 \leq|\alpha| \leq M} G_{|\alpha|}^{(\alpha)}(\varphi) \sum_{\ell=1}^{j+1} \partial_{\eta}^{\alpha} m_{\ell} a_{j+1-\ell}, \\
\widetilde{E}_{M+2}=\sum_{0 \leq|\alpha| \leq M} \partial_{\eta}^{\alpha} m_{M+2}^{\sharp}\left(G_{|\alpha|}^{(\alpha)}(\varphi) a+\sum_{\nu=1}^{M+|\alpha|} h^{\nu} a_{\nu}^{(\alpha)}\right) \\
+\sum_{0 \leq|\alpha| \leq M} \sum_{\nu=M+1}^{2 M+|\alpha|} h^{\nu-M-1} \sum_{\ell=0}^{\nu+1} \partial_{\eta}^{\alpha} m_{\ell} a_{\nu+2-\ell}^{(\alpha)} .
\end{gathered}
$$

We can expand the function $A_{M}$ as follows:

$$
\begin{gathered}
A_{M}=\left(\left(\partial_{t} \varphi\right)^{2}+\partial_{y_{1}} \varphi-\varrho^{2}+g_{M}\left(m_{0}, \varphi\right)\right) a \\
-\sum_{j=0}^{M} h^{j+1}\left(2 i \partial_{t} \varphi \partial_{t} a_{j}+i \partial_{y_{1}} a_{j}+i \partial_{t}^{2} \varphi a_{j}+\partial_{t}^{2} a_{j-1}-E_{j}^{(M)}\right) \\
-h^{M+2} \partial_{t}^{2} a_{M}+h^{M+2} \widetilde{E}_{M+2} .
\end{gathered}
$$

We would like to determine the functions $\varphi$ and $a_{j}, j=0,1, \ldots, M$, so that $A_{M}=\mathcal{O}\left(t^{M}\right)+$ $\mathcal{O}\left(h^{M+2}\right)$. To this end, we let the function $\varphi$ satisfy the following eikonal equation $\bmod \mathcal{O}\left(t^{M}\right)$ :

$$
\left(\partial_{t} \varphi\right)^{2}+\partial_{y_{1}} \varphi-\rho^{2}+g_{M}\left(m_{0}, \varphi\right)=t^{M} R_{M}
$$

where the function $R_{M}$ is smooth up to $t=0$. The functions $a_{j}$ satisfy the transport equations $\bmod \mathcal{O}\left(t^{M}\right)$ :

$$
2 i \partial_{t} \varphi \partial_{t} a_{j}+i \partial_{y_{1}} a_{j}+i \partial_{t}^{2} \varphi a_{j}+\partial_{t}^{2} a_{j-1}-E_{j}^{(M)}=t^{M} Q_{M}^{(j)}, \quad 0 \leq j \leq M,
$$

where $a_{-1}=0$ and the functions $Q_{M}^{(j)}$ are smooth up to $t=0$. Then we get

$$
A_{M}=t^{M} B_{M}+h^{M} C_{M}
$$

where

$$
B_{M}=R_{M} a-\sum_{j=0}^{M} h^{j+1} Q_{M}^{(j)}
$$




$$
C_{M}=-h^{2} \partial_{t}^{2} a_{M}+h^{2} \widetilde{E}_{M+2} .
$$

We will first solve equation (2.6). We will be looking for $\varphi$ in the form

$$
\varphi=\sum_{k=1}^{M} t^{k} \varphi_{k}
$$

with functions $\varphi_{k}$ independent of $t$. We have

$$
\begin{gathered}
\left(\partial_{t} \varphi\right)^{2}=\sum_{K=0}^{2 M-2} t^{K} \sum_{k+j=K}(k+1)(j+1) \varphi_{k+1} \varphi_{j+1}, \\
\partial_{y_{1}} \varphi=\sum_{K=1}^{M} t^{K} \partial_{y_{1}} \varphi_{K}, \\
G_{|\alpha|}^{(\alpha)}(\varphi)=\sum_{k=|\alpha|}^{M|\alpha|} t^{k} \mathcal{V}_{k}^{(\alpha)}\left(\varphi_{1}, \ldots, \varphi_{k}\right)
\end{gathered}
$$

where $\mathcal{V}_{0}^{(0)}=1$, while for $|\alpha| \geq 1, k \geq|\alpha|$ we have

$$
\mathcal{V}_{k}^{(\alpha)}=\sum_{k_{j} \geq 1, k_{1}+\ldots+k_{|\alpha|}=k} \sum_{\left|\gamma_{1}\right|=\ldots=\left|\gamma_{|\alpha|}\right|=1} c_{k_{1}, \ldots, k_{|\alpha|}, \gamma_{1}, \ldots, \gamma_{|\alpha|}} \prod_{j=1}^{|\alpha|} \partial_{y}^{\gamma_{j}} \varphi_{k_{j}}
$$

where the coefficients $c_{k_{1}, \ldots, k_{|\alpha|}, \gamma_{1}, \ldots, \gamma_{|\alpha|}}$ are real constants. We now expand the function $m_{0}$ as

$$
m_{0}=\sum_{k=1}^{M-1} t^{k} m_{k, 0}+t^{M} \widetilde{m}_{M, 0}
$$

where the functions $m_{k, 0}, k=1, \ldots, M-1$, are independent of $t$, while the function $\widetilde{m}_{M, 0}$ is smooth up to $t=0$. Hence

$$
g_{M}\left(m_{0}, \varphi\right)=\sum_{K=1}^{M-1} t^{K} \sum_{0 \leq|\alpha| \leq K-1} \sum_{\nu=1}^{K-|\alpha|} \partial_{\eta}^{\alpha} m_{\nu, 0} \mathcal{V}_{K-\nu}^{(\alpha)}+t^{M} \widetilde{g}_{M}
$$

where

$$
\begin{gathered}
\widetilde{g}_{M}=\sum_{0 \leq|\alpha| \leq M} \partial_{\eta}^{\alpha} \widetilde{m}_{M, 0} G_{|\alpha|}^{(\alpha)}(\varphi)+\sum_{0 \leq|\alpha| \leq M} t^{-1} \partial_{\eta}^{\alpha} m_{0} \sum_{k=M-1}^{M|\alpha|} t^{k-M+1} \mathcal{V}_{k}^{(\alpha)} \\
+\sum_{K=M}^{2 M-1} t^{K-M} \sum_{0 \leq|\alpha| \leq K-1} \sum_{\nu=1}^{K-|\alpha|} \partial_{\eta}^{\alpha} m_{\nu, 0} \mathcal{V}_{K-\nu}^{(\alpha)} .
\end{gathered}
$$

Inserting the above identities into equation (2.6) and comparing the coefficients of all powers $t^{K}, 0 \leq K \leq M-1$, we get the following relations for the functions $\varphi_{k}$ :

$$
\begin{gathered}
\varphi_{1}^{2}=\varrho^{2} \\
\sum_{k+j=K}(k+1)(j+1) \varphi_{k+1} \varphi_{j+1}+\partial_{y_{1}} \varphi_{K}+m_{K, 0} \\
+\sum_{1 \leq|\alpha| \leq K-1} \sum_{\nu=1}^{K-|\alpha|} \partial_{\eta}^{\alpha} m_{\nu, 0} \mathcal{V}_{K-\nu}^{(\alpha)}\left(\varphi_{1}, \ldots, \varphi_{K-\nu}\right)=0,
\end{gathered}
$$


$1 \leq K \leq M-1$, where the second sum is zero if $K=1$. Then equation (2.6) is satisfied with

$$
R_{M}=\widetilde{g}_{M}+\sum_{K=M}^{2 M-2} t^{K-M} \sum_{k+j=K}(k+1)(j+1) \varphi_{k+1} \varphi_{j+1}+\partial_{y_{1}} \varphi_{M} .
$$

We take $\varphi_{1}=\varrho$. Then, given $\varphi_{1}, \ldots, \varphi_{K}$, we can find $\varphi_{K+1}$ from equation (2) in a unique way. Thus we can find all functions $\varphi_{1}, \ldots, \varphi_{M}$. In what follows in this section, given a function $p>0$ on $T^{*} Y$ and $k \in \mathbf{R}$, we will denote by $S^{k}(p)$ the set of all functions $a \in C^{\infty}\left(T^{*} Y\right)$ satisfying $\partial_{y}^{\alpha} a=\mathcal{O}_{\alpha}\left(p^{k}\right)$ for all multi-indices $\alpha$.

Lemma 2.3. For all integers $k \geq 1$ we have $\varphi_{k} \in S^{3-2 k}(|\varrho|)$ and

$$
\left|\operatorname{Im} \partial_{y}^{\alpha} \varphi_{k}\right| \leq C_{k, \alpha}|\varrho|^{2-2 k} \operatorname{Im} \varrho .
$$

Moreover, if $0<t \leq 2 \delta|\varrho|^{2}$ with a constant $\delta>0$ small enough, we have

$$
\operatorname{Im} \varphi \geq t \operatorname{Im} \varrho / 2 .
$$

We also have that the functions $\varphi_{k}$ and $\varphi_{k+1}+\frac{m_{k, 0}}{2(k+1) \varrho}$ are independent of all $m_{\ell, 0}$ with $\ell \geq k$.

Proof. We will proceed by induction in $k$. Clearly, $\varphi_{1} \in S^{1}(|\varrho|)$. Suppose now that $\varphi_{k} \in$ $S^{3-2 k}(|\varrho|)$ for $1 \leq k \leq K$. This implies

$$
\begin{gathered}
\varphi_{k+1} \varphi_{K-k+1} \in S^{2-2 K}(|\varrho|), \quad 1 \leq k \leq K-1, \\
\partial_{y_{1}} \varphi_{K} \in S^{3-2 K}(|\varrho|), \\
\mathcal{V}_{K-\nu}^{(\alpha)}\left(\varphi_{1}, \ldots, \varphi_{K-\nu}\right) \in S^{3|\alpha|+2 \nu-2 K}(|\varrho|) .
\end{gathered}
$$

Therefore, by equation (2) we conclude that $\varrho \varphi_{K+1} \in S^{2-2 K}(|\varrho|)$, which implies $\varphi_{K+1} \in$ $S^{1-2 K}(|\varrho|)$ as desired. The bound (2.10) can also been easily proved by induction in $k$. Indeed, differentiating equation (2) allows to express $\partial_{y}^{\alpha} \varphi_{K+1}$ in terms of the functions $m_{1,0}, \ldots, m_{K, 0}$, $\varphi_{1}, \ldots, \varphi_{K}$ and their derivatives. Thus we can bound $\left|\operatorname{Im} \partial_{y}^{\alpha} \varphi_{K+1}\right|$ by using that by assumption we have

$$
\operatorname{Im} \partial_{y}^{\alpha} m_{k, 0}=\mathcal{O}(|\mu|)=\mathcal{O}(|\varrho| \operatorname{Im} \varrho)
$$

together with the inequality

$$
\left|\operatorname{Im}\left(z_{1} \ldots z_{k}\right)\right| \leq C_{k}\left|z_{1}\right| \ldots\left|z_{k}\right| \sum_{j=1}^{k} \frac{\left|\operatorname{Im} z_{j}\right|}{\left|z_{j}\right|} .
$$

The bound (2.11) follows from (2.10). We have, for $0<t \leq 2 \delta|\varrho|^{2}$,

$$
\begin{gathered}
\operatorname{Im} \varphi=\sum_{k=1}^{M} t^{k} \operatorname{Im} \varphi_{k} \geq t \operatorname{Im} \varrho\left(1-C \sum_{k=1}^{M-1} t^{k}|\varrho|^{-2 k}\right) \\
\geq t \operatorname{Im} \varrho(1-\mathcal{O}(\delta)) \geq t \operatorname{Im} \varrho / 2
\end{gathered}
$$

provided $\delta$ is taken small enough. The last assertion also follows by induction in $k$. Indeed, by equation (2) we can express the function $\varphi_{K+1}+\frac{m_{K, 0}}{2(K+1) \varrho}$ in terms of the functions $m_{1,0}, \ldots, m_{K-1,0}, \varphi_{1}, \ldots, \varphi_{K}$ and their derivatives. Therefore, it is independent of all $m_{\ell, 0}$ with $\ell \geq K$, provided so are $\varphi_{k}$ with $k \leq K$.

The above lemma implies the following 
Lemma 2.4. For all $|\beta| \geq 0,0 \leq k \leq|\beta|$, we have the identity

$$
G_{k}^{(\beta)}(\varphi)=\sum_{\nu=k}^{k M} t^{\nu} \Theta_{\nu}^{(k, \beta)}\left(m_{1,0}, \ldots, m_{\nu-k, 0}\right)
$$

where the function $\Theta_{\nu}^{(k, \beta)}$ is independent of $t$ and all $m_{\ell, 0}$ with $\ell \geq \nu-k+1$. Moreover, $\Theta_{0}^{(0,0)}=1$, $\Theta_{\nu}^{(0, \beta)}=0$ for all $\nu \geq 0$ if $|\beta| \geq 1$, while for all $|\beta| \geq 1,1 \leq k \leq|\beta|$, we have $\Theta_{\nu}^{(k, \beta)} \in S^{-2 \nu}(|\varrho|)$.

Proof. It is clear from the definition of the function $G_{k}^{(\beta)}(\varphi)$ that we have (2.12) with $\Theta_{\nu}^{(k, \beta)}$ being a linear combination of functions of the form

$$
\prod_{j=1}^{k} \partial_{y}^{\gamma_{j}} \varphi_{\nu_{j}}
$$

where $1 \leq \nu_{j} \leq M$ are integers such that $\nu_{1}+\ldots+\nu_{k}=\nu$. Hence $\nu$ must satisfy $k \leq \nu \leq k M$ and each $\nu_{j}$ must satisfy $\nu_{j} \leq \nu-k+1$. Therefore, the lemma is an immediate consequence of Lemma 2.3.

We will now be looking for solutions to the equations (2.7) in the form

$$
a_{j}=\sum_{k=0}^{M} t^{k} a_{k, j} .
$$

We have

$$
\begin{gathered}
\partial_{t} \varphi \partial_{t} a_{j}=\sum_{k=0}^{2 M-2} t^{k} \sum_{\nu=0}^{k}(k-\nu+1)(\nu+1) \varphi_{k-\nu+1} a_{\nu+1, j}, \\
\partial_{y_{1}} a_{j}=\sum_{k=0}^{M} t^{k} \partial_{y_{1}} a_{k, j}, \\
\partial_{t}^{2} \varphi a_{j}=\sum_{k=0}^{2 M-2} t^{k} \sum_{\nu=0}^{k}(k-\nu+1)(k-\nu+2) \varphi_{k-\nu+2} a_{\nu, j}, \\
\partial_{t}^{2} a_{j-1}=\sum_{k=0}^{M-2} t^{k}(k+1)(k+2) a_{k+2, j-1} .
\end{gathered}
$$

We will now use Lemma 2.4 to prove the following

Lemma 2.5. We have the identity

$$
E_{j}^{(M)}=\sum_{k=0}^{M(M+2)} t^{k} E_{k, j}^{(M)}
$$

with functions $E_{k, j}^{(M)}$ independent of $t$ having the form

$$
E_{k, j}^{(M)}=\sum_{\nu=0}^{k} \sum_{\ell=0}^{j} \sum_{|\gamma| \leq M} \Psi_{\nu, \ell, \gamma}^{(k, j)} \partial_{y}^{\gamma} a_{\nu, \ell}
$$

where the function $\Psi_{\nu, \ell, \gamma}^{(k, j)} \in S^{-2(k-\nu)}(|\varrho|)$ depends only on $m_{k^{\prime}, j^{\prime}}$ with $k^{\prime} \leq k, j^{\prime} \leq j+1$. 
Proof. Using (2.12) we expand the function $a_{\nu}^{(\alpha)}$ as

$$
a_{\nu}^{(\alpha)}=\sum_{k=0}^{M(|\alpha|+1)} t^{k} a_{k, \nu}^{(\alpha)}
$$

where

Hence

$$
a_{k, \nu}^{(\alpha)}=\sum_{\nu^{\prime}=(\nu-|\alpha|)_{+}}^{\nu-1} \sum_{k^{\prime}=0}^{k+\nu-\nu^{\prime}-|\alpha|} \sum_{|\beta| \leq \nu-\nu^{\prime}} c_{\alpha, \beta} \Theta_{k-k^{\prime}}^{\left(|\alpha|-\nu+\nu^{\prime}, \alpha-\beta\right)} \partial_{y}^{\beta} a_{k^{\prime}, \nu^{\prime}}
$$

where

$$
\begin{gathered}
\sum_{\ell=0}^{j} \partial_{\eta}^{\alpha} m_{\ell} a_{j+1-\ell}^{(\alpha)}=\sum_{k=0}^{M(|\alpha|+2)} t^{k} \sum_{k_{1}=0}^{k} \sum_{j_{1}=1}^{j+1} \partial_{\eta}^{\alpha} m_{k-k_{1}, j+1-j_{1}} a_{k_{1}, j_{1}}^{(\alpha)} \\
=\sum_{k=0}^{M(|\alpha|+2)} t^{k} \sum_{k^{\prime}=0}^{k} \sum_{j^{\prime}=0}^{j} \sum_{|\beta| \leq|\alpha|} F_{k^{\prime}, j^{\prime}, \beta}^{(k, j)} \partial_{y}^{\beta} a_{k^{\prime}, j^{\prime}}
\end{gathered}
$$

$$
F_{k^{\prime}, j^{\prime}, \beta}^{(k, j, \alpha)}=c_{\alpha, \beta} \sum_{\left(k_{1}, j_{1}\right) \in \Xi\left(k, j, k^{\prime}, j^{\prime}, \alpha, \beta\right)} \partial_{\eta}^{\alpha} m_{k-k_{1}, j+1-j_{1}} \Theta_{k_{1}-k^{\prime}}^{\left(|\alpha|-j_{1}+j^{\prime}, \alpha-\beta\right)}
$$

where $\Xi\left(k, j, k^{\prime}, j^{\prime}, \alpha, \beta\right)$ denotes the set of all integers $\left(k_{1}, j_{1}\right)$ satisfying $0 \leq k_{1} \leq k, 1 \leq j_{1} \leq$ $j+1, j^{\prime}+|\beta| \leq j_{1} \leq j^{\prime}+|\alpha|, k_{1}+j_{1} \geq k^{\prime}+j^{\prime}+|\alpha|$. Clearly, if this set is empty, the above sum is zero. By Lemma 2.4 we get

$$
F_{k^{\prime}, j^{\prime}, \beta}^{(k, j, \alpha)} \in S^{-2\left(k-k^{\prime}\right)}(|\varrho|) .
$$

Furthermore, we have

$$
\sum_{\ell=1}^{j+1} \partial_{\eta}^{\alpha} m_{\ell} a_{j+1-\ell}=\sum_{k=0}^{2 M} t^{k} \sum_{k^{\prime}=0}^{k} \sum_{j^{\prime}=0}^{j} \partial_{\eta}^{\alpha} m_{k-k^{\prime}, j+1-j^{\prime}} a_{k^{\prime}, j^{\prime}} .
$$

Hence

$$
\begin{aligned}
G_{|\alpha|}^{(\alpha)}(\varphi) \sum_{\ell=1}^{j+1} \partial_{\eta}^{\alpha} m_{\ell} a_{j+1-\ell} & =\sum_{k=0}^{M(|\alpha|+2)} t^{k} \sum_{k_{1}=0}^{k-|\alpha|} \sum_{k^{\prime}=0}^{k_{1}} \sum_{j^{\prime}=0}^{j} \mathcal{V}_{k-k_{1}}^{(\alpha)} \partial_{\eta}^{\alpha} m_{k_{1}-k^{\prime}, j+1-j^{\prime}} a_{k^{\prime}, j^{\prime}} \\
= & \sum_{k=0}^{M(|\alpha|+2)} t^{k} \sum_{k^{\prime}=0}^{k-|\alpha|} \sum_{j^{\prime}=0}^{j} \widetilde{F}_{k^{\prime}, j^{\prime}}^{(k, j, \alpha)} a_{k^{\prime}, j^{\prime}}
\end{aligned}
$$

where

$$
\widetilde{F}_{k^{\prime}, j^{\prime}}^{(k, j)}=\sum_{k_{1}=k^{\prime}}^{k-|\alpha|} \mathcal{V}_{k-k_{1}}^{(\alpha)} \partial_{\eta}^{\alpha} m_{k_{1}-k^{\prime}, j+1-j^{\prime}} \in S^{-2\left(k-k^{\prime}\right)}(|\varrho|) .
$$

It follows from the above identities that the desired expansion of the function $E_{j}^{(M)}$ holds with

$$
\begin{aligned}
\Psi_{\nu, \ell, \gamma}^{(k, j)} & =\sum_{|\gamma| \leq|\alpha| \leq M} F_{\nu, \ell, \gamma}^{(k, j, \alpha)} \in S^{-2(k-\nu)}(|\varrho|), \quad|\gamma| \geq 1, \\
\Psi_{\nu, \ell, 0}^{(k, j)} & =\sum_{0 \leq|\alpha| \leq M}\left(F_{\nu, \ell, 0}^{(k, j, \alpha)}+\widetilde{F}_{\nu, \ell}^{(k, j, \alpha)}\right) \in S^{-2(k-\nu)}(|\varrho|) .
\end{aligned}
$$


Inserting the above identities into equations (2.7) and comparing the coefficients of all powers $t^{k}, 0 \leq k \leq M-1$, we get the following relations for the functions $a_{k, j}$ :

$$
\begin{gathered}
2 i \sum_{\nu=0}^{k}(k-\nu+1)(\nu+1) \varphi_{k-\nu+1} a_{\nu+1, j}+i \sum_{\nu=0}^{k}(k-\nu+1)(k-\nu+2) \varphi_{k-\nu+2} a_{\nu, j} \\
+i \partial_{y_{1}} a_{k, j}+(k+1)(k+2) a_{k+2, j-1}=E_{k, j}^{(M)}
\end{gathered}
$$

and $a_{0,0}=\phi\left(\eta_{1}\right), a_{0, j}=0, j \geq 1, a_{k,-1}=0, k \geq 0$. Since the function $E_{k, j}^{(M)}$ depends only on $a_{k^{\prime}, j^{\prime}}$ with $k^{\prime} \leq k, j^{\prime} \leq j$, it is easy to see that we can determine all $a_{k, j}$ from equations (2). Observe also that $\operatorname{supp}_{\eta_{1}} a_{k, j} \equiv \operatorname{supp} \phi\left(\eta_{1}\right)$. Then the equations (2.7) are satisfied with

$$
\begin{gathered}
Q_{M}^{(j)}=2 i \sum_{k=M}^{2 M-2} t^{k-M} \sum_{\nu=0}^{M-1}(k-\nu+1)(\nu+1) \varphi_{k-\nu+1} a_{\nu+1, j}+i \partial_{y_{1}} a_{M, j} \\
+i \sum_{k=M}^{2 M-2} t^{k-M} \sum_{\nu=0}^{M}(k-\nu+1)(k-\nu+2) \varphi_{k-\nu+2} a_{\nu, j} \\
\quad-\sum_{k=M}^{M(M+2)} t^{k-M} \sum_{\nu=0}^{M} \sum_{\ell=0}^{j} \sum_{|\gamma| \leq M} \Psi_{\nu, \ell, \gamma}^{(k, j)} \partial_{y}^{\gamma} a_{\nu, \ell} .
\end{gathered}
$$

We will now prove the following

Lemma 2.6. For all $k \geq 0, j \geq 0$, we have $a_{k, j} \in S^{-2 k-3 j}(|\varrho|)$. Moreover, the function

$$
a_{k, j}+\frac{(k+j) !}{k !} \frac{\phi m_{k+j, 0}}{(-2 i \varrho)^{j+2}}
$$

depends only on $m_{\nu, 0}$ with $\nu \leq k+j-1$ and $m_{\nu, \ell}$ with $1 \leq \ell \leq j+1, \nu+\ell \leq k+j$.

Proof. We will proceed by induction. Clearly, the first assertion is trivial for $k=0$ and all $j \geq 0$. Suppose that it is true for all $j \leq J-1, k \geq 0$, and for $j=J, k \leq K$. We have to show that it is true for $j=J$ and $k=K+1$. In view of Lemmas 2.3 and 2.5 we have

$$
\begin{gathered}
\varphi_{k-\nu+1} a_{\nu+1, j} \in S^{-2 k-3 j-1}(|\varrho|), \quad 0 \leq \nu \leq k-1, k=K, j=J, \\
\varphi_{k-\nu+2} a_{\nu, j} \in S^{-2 k-3 j-1}(|\varrho|), \quad 0 \leq \nu \leq k, k=K, j=J, \\
\partial_{y_{1}} a_{k, j} \in S^{-2 k-3 j}(|\varrho|), \quad k=K, j=J \\
a_{k+2, j-1} \in S^{-2 k-3 j-1}(|\varrho|), \quad k=K, j=J, \\
E_{k, j}^{(M)} \in S^{-2 k-3 j}(|\varrho|), \quad k=K, j=J .
\end{gathered}
$$

Therefore, by equation (2) we conclude $\varrho a_{K+1, J} \in S^{-2 K-3 J-1}(|\varrho|)$, which implies $a_{K+1, J} \in$ $S^{-2 K-3 J-2}(|\varrho|)$ as desired.

We turn now to the proof of the second assertion. We will first prove it for $j=0$ and all $k \geq 0$ by induction in $k$. It is trivially fulfilled for $k=0$. Suppose it is fulfilled for all $k \leq K$. We have to show it is fulfilled for $k=K+1$. By Lemma 2.5 we have that $E_{K, 0}^{(M)}$ depends on $m_{\nu, \ell}$ with $0 \leq \ell \leq 1, \nu \leq K$. Therefore, by equation (2) with $j=0, k=K$, we get that

$$
2 i(K+1) \varrho a_{K+1,0}+i(K+1)(K+2) \varphi_{K+2} a_{0,0}
$$

depends on $m_{\nu, \ell}$ with $0 \leq \ell \leq 1,0 \leq \nu \leq K$. We now use the last assertion in Lemma 2.3 to conclude that

$$
a_{K+1,0}-(2 \varrho)^{-2} m_{K+1,0} \phi
$$


depends on $m_{\nu, \ell}$ with $0 \leq \ell \leq 1,0 \leq \nu \leq K$, as desired.

We will now proceed by induction in both $j$ and $k$. The assertion is trivially fulfilled for $k=0$. Suppose it is true for all $j \leq J-1, k \geq 0$ and for $j=J, k \leq K$ with some integers $K \geq 0$, $J \geq 1$. We will prove it for $j=J$ and $k=K+1$. To this end we will use equation (2) with $k=K$ and $j=J$. By Lemma 2.5 we have that $E_{K, J}^{(M)}$ depends on $m_{\nu, 0}$ with $\nu \leq K+J$ and $m_{\nu, \ell}$ with $1 \leq \ell \leq J+1, \nu+\ell \leq K+J$. Therefore, we get that so does the function

$$
2 i \varrho(K+1) a_{K+1, J}+(K+1)(K+2) a_{K+2, J-1} .
$$

On the other hand, since the assertion is supposed to be fulfilled for $j=J-1$ and all $k$, we have that the function

$$
(K+2) a_{K+2, J-1}+\frac{(K+1+J) !}{(K+1) !} \frac{\phi m_{K+1+J, 0}}{(-2 i \varrho)^{J+1}}
$$

depends only on $m_{\nu, 0}$ with $\nu \leq K+J$ and $m_{\nu, \ell}$ with $1 \leq \ell \leq J, \nu+\ell \leq K+1+J$. Thus we conclude that the function

$$
a_{K+1, J}+\frac{(K+1+J) !}{(K+1) !} \frac{\phi m_{K+1+J, 0}}{(-2 i \varrho)^{J+2}}
$$

depends only on $m_{\nu, 0}$ with $\nu \leq K+J$ and $m_{\nu, \ell}$ with $1 \leq \ell \leq J+1, \nu+\ell \leq K+1+J$, as desired.

It is clear from the equations (2) that the functions $a_{k, j}$ are well-defined for all $\mu \neq 0$ because so is the function $\varrho^{-1}$. The condition $|\mu| \geq h^{2 / 3-\epsilon}$ is only used to show that the above construction provides a parametrix for the boundary value problem (2.3) (see the proof of Proposition 2.8 below). It is also possible to bound the derivatives of $a_{k, j}$ with repspect to the variable $\eta$ uniformly in $\mu$. Thus, although we do not need this information in the analysis that follows, for some values of $\mu$ we can describe completely the class of symbols the functions $a_{k, j}$ belong to. For example, we have the following

Lemma 2.7. Let $|\mu| \geq h^{2 / 3}$. Then we have $a_{k, j} \in S_{0,2 / 3}^{2 k / 3+j, 0}$.

Proof. In the same way as above one can show that the functions $a_{k, j}$ satisfy the bounds

$$
\left|\partial_{y}^{\alpha} \partial_{\eta}^{\beta} a_{k, j}\right| \leq C_{\alpha, \beta}|\varrho|^{-2 k-3 j-2 \beta_{1}} \leq C_{\alpha, \beta}|\mu|^{-k-3 j / 2-\beta_{1}}
$$

for all multi-indices $\alpha$ and $\beta=\left(\beta_{1}, \ldots\right)$, which clearly imply the lemma.

We will now show that $\widetilde{u}$ provides the desired parametrix. Recall that $\widetilde{u}$ depends on the parameter $M$. We have the following

Proposition 2.8. Let $h^{2 / 3-\epsilon} \leq|\mu| \leq 1$. Then there is $M_{0}>0$ depending on $\epsilon$ such that for $M \geq M_{0}$ we have the bounds

$$
\begin{gathered}
\|\widetilde{u}\|_{L^{2}((0,1) \times Y)} \lesssim h^{-d}\|f\|_{L^{2}(Y)}, \\
\left\|P_{0}(h, \mu) \widetilde{u}\right\|_{L^{2}((0,1) \times Y)} \lesssim h^{\epsilon M / 2}\|f\|_{L^{2}(Y)} .
\end{gathered}
$$

Proof. We will use the identity (2.4). Observe first that by Lemma 2.3 we have $\partial_{t} \varphi, \varphi / t \in$ $S^{1}(|\varrho|)$ and

$$
\operatorname{Im} \varphi \geq t \operatorname{Im} \varrho / 2 \geq \frac{t|\mu|}{4|\varrho|}
$$


as long as $0<t \leq 2 \delta|\varrho|^{2}$. Thus, by Lemma 2.2 we get

$$
\left|\partial_{y}^{\alpha}\left(e^{i \varphi / h}\right)\right| \lesssim\left(\frac{t}{h}\right)^{|\alpha|} \exp \left(-\frac{t|\mu|}{4 h|\varrho|}\right) .
$$

In particular (2.16) implies

$$
\left|\partial_{y}^{\alpha}\left(e^{i \varphi / h}\right)\right| \lesssim \exp \left(-h^{-3 \epsilon / 2} / 5\right)
$$

for $h^{\epsilon} \leq t \leq 2 h^{\epsilon}$ or $\delta|\varrho|^{2} \leq t \leq 2 \delta|\varrho|^{2}$. Next by Lemma 2.6 we have, with any $\nu \geq 0$,

$$
t^{k-\nu} a_{k, j} \in S^{-2 \nu-3 j}(|\varrho|) \subset S^{-\nu-3 j / 2}(|\mu|) \subset S^{-2 \nu / 3-j}(h)
$$

as long as $0<t \leq 2 \delta|\varrho|^{2}$. Hence

$$
t^{k-\nu} h^{j} a_{k, j} \in S^{-2 \nu / 3}(h)
$$

which implies

$$
\partial_{t}^{\nu} a \in S^{-2 \nu / 3}(h), \quad \nu \geq 0 .
$$

It follows from (2.19) that $\partial_{y}^{\alpha} A_{M}^{\sharp}=\mathcal{O}_{\alpha}(1)$ which together with (2.17) imply

$$
\left|\partial_{y}^{\alpha}\left(e^{i \varphi / h} A_{M}^{\sharp}\right)\right| \lesssim \exp \left(-h^{-3 \epsilon / 2} / 5\right) .
$$

This bound together with (2.1) yield

$$
\left\|\mathrm{Op}_{h}\left(e^{i \varphi / h} A_{M}^{\sharp}\right)\right\|_{L^{2}(Y) \rightarrow L^{2}(Y)} \lesssim \exp \left(-h^{-3 \epsilon / 2} / 5\right) .
$$

By (2.16) and (2.19) with $\nu=0$ we also have

$$
\left|\partial_{y}^{\alpha}\left(e^{i \varphi / h} \Phi_{\epsilon, \delta} a\right)\right| \lesssim h^{-(1-\epsilon)|\alpha|} .
$$

Therefore by Proposition 2.1 we get

$$
\left\|\mathcal{E}_{M}\right\|_{L^{2}(Y) \rightarrow L^{2}(Y)} \lesssim h^{\epsilon M / 2}
$$

for $M$ big enough, while the bound (2.1) yields

$$
\left\|\mathrm{Op}_{h}\left(e^{i \varphi / h} \Phi_{\epsilon, \delta} a\right)\right\|_{L^{2}(Y) \rightarrow L^{2}(Y)} \lesssim h^{-d} .
$$

To bound the norm of the first operator in the right-hand side of (2.4) we will make use of (2.8). Using (2.18) together with Lemmas 2.3 and 2.5 it is not hard to check that the functions $B_{M}$ and $C_{M}$ belong to the spaces $S^{-2 M}(|\varrho|)$ and $S^{-3 M}(|\varrho|)$, respectively, uniformly in $h$ and in $t \in \operatorname{supp} \Phi_{\epsilon, \delta}$. This fact together with (2.16) lead to the bounds

$$
\begin{aligned}
\left|\partial_{y}^{\alpha}\left(e^{i \varphi / h} \Phi_{\epsilon, \delta} A_{M}\right)\right| & \leq t^{M}\left|\partial_{y}^{\alpha}\left(e^{i \varphi / h} \Phi_{\epsilon, \delta} B_{M}\right)\right|+h^{M}\left|\partial_{y}^{\alpha}\left(e^{i \varphi / h} \Phi_{\epsilon, \delta} C_{M}\right)\right| \\
\lesssim & h^{-|\alpha|}\left(t^{M}|\varrho|^{-2 M} e^{-\frac{t|\mu|}{4 h|\varrho|}}+h^{M}|\varrho|^{-3 M}\right) \\
& \lesssim h^{M-|\alpha|}\left(|\varrho|^{-M}|\mu|^{-M}+|\varrho|^{-3 M}\right) \\
& \lesssim h^{M-|\alpha|}|\mu|^{-3 M / 2} \lesssim h^{3 \epsilon M / 2-|\alpha|} .
\end{aligned}
$$

Thus by (2.1) we get

$$
\left\|\operatorname{Op}_{h}\left(e^{i \varphi / h} \Phi_{\epsilon, \delta} A_{M}\right)\right\|_{L^{2}(Y) \rightarrow L^{2}(Y)} \lesssim h^{\epsilon M} .
$$


Combining (2.20), (2.21) and (2.23) we obtain, for $M$ big enough,

$$
\left\|P_{0}(h, \mu) \widetilde{u}\right\|_{L^{2}(Y)}^{2} \lesssim h^{\epsilon M}\|f\|_{L^{2}(Y)}^{2}
$$

uniformly in $t$. Integrating (2.24) with respect to $t$ we get (2.15). The bound (2.14) follows in the same way from (2.22).

Define the operator $\tilde{N}(h, \mu)$ by $\tilde{N} f:=\left.\mathcal{D}_{t} \widetilde{u}\right|_{t=0}$. We have

$$
\tilde{N}=\operatorname{Op}_{h}\left(\left.a \partial_{t} \varphi\right|_{t=0}-\left.i h \partial_{t} a\right|_{t=0}\right)=\operatorname{Op}_{h}\left(\varrho \phi-i \sum_{j=0}^{M} h^{j+1} a_{1, j}\right) .
$$

Let $s, k \geq 0$ be arbitrary integers such that $k \leq 3 s+2$ and take $M \gg s$. Set

$$
\widetilde{N}_{s, k}=\operatorname{Op}_{h}\left(\varrho^{k+1} \phi-i \sum_{j=0}^{s-1} h^{j+1} \varrho^{k} a_{1, j}\right)
$$

where the sum is zero if $s=0$. Let $\phi_{1} \in C_{0}^{\infty}$ be such that $\phi_{1}=1$ on $\operatorname{supp} \phi$. Clearly, $\left(1-\phi_{1}\left(\eta_{1}\right)\right) a_{1, j} \equiv 0$.

Lemma 2.9. For $|\mu| \geq h^{2 / 3}$ we have the estimate

$$
\left\|\widetilde{N}(h, \mu) \mathrm{Op}_{h}\left(\phi_{1} \varrho^{k}\right)-\widetilde{N}_{s, k}\right\|_{L^{2}(Y) \rightarrow L^{2}(Y)} \lesssim h^{s+1}|\mu|^{-(3 s+2-k) / 2} .
$$

Proof. It follows from Lemma 2.6 that

$$
\varrho^{k} a_{1, j} \in S^{-3 j-2+k}(|\varrho|) \subset S^{-(3 j+2-k) / 2}(|\mu|), \quad j \geq s .
$$

Therefore, by (2.1) we get

$$
\left\|\mathrm{Op}_{h}\left(\varrho^{k} a_{1, j}\right)\right\|_{L^{2}(Y) \rightarrow L^{2}(Y)} \lesssim|\mu|^{-(3 j+2-k) / 2}, \quad j \geq s,
$$

which clearly implies (2.25).

\section{Parametrix COnstruction in the GLANCing Region}

We will use the parametrix from the previous section to construct a parametrix for the boundary value problem (1.2) with $f$ replaced by $\operatorname{Op}_{h}(\chi) f$. Then using our parametrix we will prove the following

Theorem 3.1. Let $|\operatorname{Im} z| \geq h^{2 / 3-\epsilon}, 0<\epsilon \ll 1$. Then, for every integer $s \geq 0$ there is an operator

$$
\mathcal{A}_{s}(h, z)=\mathcal{O}\left(h|\operatorname{Im} z|^{-1}\right): L^{2}(\partial X) \rightarrow L^{2}(\partial X)
$$

independent of all $n_{\ell}$ with $\ell \geq s$ such that

$$
\left\|N(h, z) \mathrm{Op}_{h}(\chi)-\widetilde{\mathrm{Op}}_{h}\left(\rho \chi+c_{s} h^{s} \rho^{-s-1} z n_{s} \chi\right)-\mathcal{A}_{s}\right\| \lesssim h^{s+1}|\operatorname{Im} z|^{-(3 s+2) / 2}
$$

where $c_{s}=0, \mathcal{A}_{s}=0$ if $s=0$, and $c_{s}=-i(-2 i)^{-s-1}$ for $s \geq 1$. Furthermore, for every integer $s \geq 1$ there are operators

$$
\mathcal{B}_{s}^{R}(h, z), \mathcal{B}_{s}^{L}(h, z)=\mathcal{O}\left(h^{-s}\right): L^{2}(\partial X) \rightarrow L^{2}(\partial X)
$$

independent of all $n_{\ell}$ with $\ell \geq 1$, and operators $\mathcal{C}_{s}^{R}(h, z), \mathcal{C}_{s}^{L}(h, z)$ independent of all $n_{\ell}$ with $\ell \geq s$ such that

$$
\left\|N(h, z) \mathcal{B}_{s}^{R}-\mathcal{C}_{s}^{R}-\mathrm{Op}_{h}\left(n_{s} \chi\right)\right\| \lesssim h|\operatorname{Im} z|^{-(2 s+1) / 2},
$$




$$
\left\|\mathcal{B}_{s}^{L} N(h, z)-\mathcal{C}_{s}^{L}-\mathrm{Op}_{h}\left(n_{s} \chi\right)\right\| \lesssim h|\operatorname{Im} z|^{-(2 s+1) / 2} .
$$

Clearly, Theorem 1.1 follows from (3.1) with $s=0$. Note that an analog of Theorem 3.1 has been proved in [12] but for $|\operatorname{Im} z| \geq h^{1 / 2-\epsilon}$ and with worse bounds in the right-hand sides of (3.1), (3.2) and (3.3).

We begin the parametrix construction by writing the Laplace-Beltrami operator in local coordinates near the boundary. Fix a point $x^{0} \in \Gamma$ and let $\mathcal{U}_{0} \subset \Gamma$ be a small open neighbourhood of $x^{0}$. Let $\left(x_{1}, x^{\prime}\right), x_{1}>0, x^{\prime} \in \mathcal{U}_{0}$, be the normal coordinates with respect to the metric $\mathcal{G}$. In these coordinates the Laplacian can be written as follows

$$
\Delta_{X}=\partial_{x_{1}}^{2}+r\left(x, \partial_{x^{\prime}}\right)+q\left(x, \partial_{x}\right)
$$

where $r\left(x, \xi^{\prime}\right)$ is homogeneous in $\xi^{\prime}$ of order two, smooth in $x$ and strictly positive for all $\xi^{\prime} \neq 0$, $q(x, \xi)=\langle q(x), \xi\rangle=q^{\sharp}(x) \xi_{1}+\left\langle q^{b}(x), \xi^{\prime}\right\rangle, q^{\sharp}$ and $q^{b}$ being smooth functions. Clearly, $r\left(0, x^{\prime}, \xi^{\prime}\right)=$ $r_{0}\left(x^{\prime}, \xi^{\prime}\right)$ is the principal symbol of the Laplace-Beltrami operator on the boundary. Introduce the function

and observe that

$$
\varphi^{\sharp}\left(x_{1}, x^{\prime}\right)=-\frac{1}{2} \int_{0}^{x_{1}} q^{\sharp}\left(\sigma, x^{\prime}\right) d \sigma
$$

$$
e^{-\varphi^{\sharp}} \Delta_{X} e^{\varphi^{\sharp}}=\partial_{x_{1}}^{2}+r\left(x, \partial_{x^{\prime}}\right)+q^{b}\left(x, \partial_{x^{\prime}}\right)+V^{\sharp}(x)
$$

with a new function $q^{b}\left(x, \xi^{\prime}\right)=\left\langle q^{b}(x), \xi^{\prime}\right\rangle, q^{b}$ and $V^{\sharp}$ being smooth functions. We now introduce a new normal variable $t=n_{0}\left(x^{\prime}\right)^{1 / 2} x_{1}, n_{0}\left(x^{\prime}\right):=n\left(0, x^{\prime}\right)>0$, and we write the operator

$$
P(h)=-h^{2} n_{0}^{-1} \Delta_{X}-1-i \operatorname{Im} z-z n_{0}^{-1}\left(n-n_{0}\right)
$$

in the coordinates $\left(t, x^{\prime}\right)$ as follows:

$$
\begin{gathered}
P_{\sharp}(h):=e^{-\varphi^{\sharp}} P(h) e^{\varphi^{\sharp}} \\
=\mathcal{D}_{t}^{2}+n_{0}^{-1} r_{0}\left(x^{\prime}, \mathcal{D}_{x^{\prime}}\right)-1-i \operatorname{Im} z-z n_{0}^{-1}\left(n\left(t n_{0}^{-1 / 2}, x^{\prime}\right)-n_{0}\right) \\
+n_{0}^{-1}\left(r\left(t n_{0}^{-1 / 2}, x^{\prime}, \mathcal{D}_{x^{\prime}}\right)-r\left(0, x^{\prime}, \mathcal{D}_{x^{\prime}}\right)\right)-i h n_{0}^{-1} q^{b}\left(t n_{0}^{-1 / 2}, x^{\prime}, \mathcal{D}_{x^{\prime}}\right)-h^{2} n_{0}^{-1} V^{\sharp}\left(t n_{0}^{-1 / 2}, x^{\prime}\right) .
\end{gathered}
$$

If $u=e^{\varphi^{\sharp}} v$, we have $\left.u\right|_{t=0}=\left.v\right|_{t=0}$ and

$$
\begin{aligned}
\left.\mathcal{D}_{\nu} u\right|_{\partial X}= & \left.\mathcal{D}_{x_{1}} u\right|_{x_{1}=0}=\left.\mathcal{D}_{x_{1}} v\right|_{x_{1}=0}+\left.\left.\mathcal{D}_{x_{1}} \varphi^{\sharp}\right|_{x_{1}=0} v\right|_{x_{1}=0} \\
& =\left.n_{0}^{1 / 2} \mathcal{D}_{t} v\right|_{t=0}+\left.\frac{i h}{2} q^{\sharp}\left(0, x^{\prime}\right) v\right|_{t=0} .
\end{aligned}
$$

If we denote

$$
\begin{gathered}
n_{k}\left(x^{\prime}\right)=\left.\partial_{\nu}^{k} n\right|_{\partial X}=\left.\partial_{x_{1}}^{k} n\left(x_{1}, x^{\prime}\right)\right|_{x_{1}=0}, \\
V_{k}^{\sharp}\left(x^{\prime}\right)=\left.\partial_{\nu}^{k} V^{\sharp}\right|_{\partial X}=\left.\partial_{x_{1}}^{k} V^{\sharp}\left(x_{1}, x^{\prime}\right)\right|_{x_{1}=0}, \\
r_{k}\left(x^{\prime}, \xi^{\prime}\right)=\left.\partial_{\nu}^{k} r\left(x, \xi^{\prime}\right)\right|_{\partial X}=\left.\partial_{x_{1}}^{k} r\left(x_{1}, x^{\prime}, \xi^{\prime}\right)\right|_{x_{1}=0}, \\
q_{k}^{b}\left(x^{\prime}, \xi^{\prime}\right)=\left.\partial_{\nu}^{k} q^{b}\left(x, \xi^{\prime}\right)\right|_{\partial X}=\left.\partial_{x_{1}}^{k} q^{b}\left(x_{1}, x^{\prime}, \xi^{\prime}\right)\right|_{x_{1}=0},
\end{gathered}
$$

we have the formal expansions

$$
\begin{gathered}
n_{0}^{-1}\left(n\left(t n_{0}^{-1 / 2}, x^{\prime}\right)-n_{0}\right) \sim \sum_{k=1}^{\infty} \frac{t^{k}}{k !} n_{k} n_{0}^{-(k+2) / 2}, \\
n_{0}^{-1} V^{\sharp}\left(t n_{0}^{-1 / 2}, x^{\prime}\right) \sim \sum_{k=0}^{\infty} \frac{t^{k}}{k !} V_{k}^{\sharp} n_{0}^{-(k+2) / 2},
\end{gathered}
$$




$$
\begin{gathered}
n_{0}^{-1}\left(r\left(t n_{0}^{-1 / 2}, x^{\prime}, \xi^{\prime}\right)-r\left(0, x^{\prime}, \xi^{\prime}\right)\right) \sim \sum_{k=1}^{\infty} \frac{t^{k}}{k !} r_{k} n_{0}^{-(k+2) / 2}, \\
n_{0}^{-1} q^{b}\left(t n_{0}^{-1 / 2}, x^{\prime}, \xi^{\prime}\right) \sim \sum_{k=0}^{\infty} \frac{t^{k}}{k !} q_{k}^{b} n_{0}^{-(k+2) / 2} .
\end{gathered}
$$

It is clear that it suffices to build the parametrix microlocally near $\Sigma$, that is, with $\chi$ supported in a small neighbourhood, $\mathcal{W}_{0} \subset T^{*} \partial X$, of a point $\zeta^{0}=\left(x^{0}, \xi^{0}\right) \in \Sigma$. Then the global parametrix is obtained by making a suitable partition of the unity on $\Sigma$ as explained in the introduction. We may suppose that $\pi \mathcal{W}_{0} \subset \mathcal{U}_{0}$, where $\pi: T^{*} \partial X \rightarrow \partial X$ denotes the projection $\pi\left(x^{\prime}, \xi^{\prime}\right)=x^{\prime}$. Let $\psi \in C_{0}^{\infty}\left(\mathcal{W}_{0}\right)$ be such that $\psi=1$ on $\operatorname{supp} \chi$. The standard calculas of $h-\Psi D O s$ (e.g see Section 7 of [2]) yield that to the function $\chi$ one can associate a linear map $\vartheta: C^{\infty}\left(T^{*} \partial X\right) \rightarrow C_{0}^{\infty}\left(\mathcal{W}_{0}\right)$ so that, if $a$ is a symbol independent of $h$, then

$$
\mathrm{Op}_{h}(a) \mathrm{Op}_{h}(\chi)-\mathrm{Op}_{h}(\chi) \mathrm{Op}_{h}(a)=\mathrm{Op}_{h}(\vartheta(a))+\mathcal{O}\left(h^{\infty}\right)
$$

where

$$
\vartheta(a) \sim \sum_{j=1}^{\infty} h^{j} \vartheta_{j}(a)
$$

with functions $\vartheta_{j}(a)$ independent of $h$ and supported in an arbitrary neighbourhood of supp $\chi$. Clearly, we can rewrite the above identity in the form

$$
\mathrm{Op}_{h}(a) \mathrm{Op}_{h}(\chi)=\mathrm{Op}_{h}(\chi) \mathrm{Op}_{h}(\psi a)+\mathrm{Op}_{h}(\vartheta(a))+\mathcal{O}\left(h^{\infty}\right)
$$

Using this we can write

$$
P_{\sharp} \mathrm{Op}_{h}(\chi)=\mathrm{Op}_{h}(\chi) \widetilde{P}_{\sharp}+\mathcal{O}\left(h^{\infty}\right)
$$

where

$$
\widetilde{P}_{\sharp}=\mathcal{D}_{t}^{2}+\left(\psi n_{0}^{-1} r_{0}\right)\left(x^{\prime}, \mathcal{D}_{x^{\prime}}\right)-1-i \operatorname{Im} z+p\left(t, x^{\prime}, \mathcal{D}_{x^{\prime}} ; h, z\right)
$$

with a function $p\left(t, x^{\prime}, \xi^{\prime} ; h, z\right)=\mathcal{O}(t+h)$ having the formal expansion

$$
p \sim \sum_{k=0}^{\infty} \sum_{j=0}^{\infty} t^{k} h^{j} p_{k, j}
$$

where the functions $p_{k, j}$ are independent of $h$ and $t$. More precisely, we have the formulas

for $k \geq 1$,

$$
p_{0,0}=0, \quad p_{k, 0}=\frac{1}{k !} \psi\left(r_{k}-z n_{k}\right) n_{0}^{-(k+2) / 2},
$$

for $k \geq 1$,

$$
\begin{gathered}
p_{0,1}=-i \psi q_{0}^{b} n_{0}^{-1}+\vartheta_{1}\left(r_{0} / n_{0}\right), \\
p_{k, 1}=-\frac{i}{k !} \psi q_{k}^{b} n_{0}^{-(k+2) / 2}+\frac{1}{k !} \vartheta_{1}\left(\left(r_{k}-z n_{k}\right) n_{0}^{-(k+2) / 2}\right),
\end{gathered}
$$

for $k \geq 1$,

$$
\begin{gathered}
p_{0,2}=-\psi V_{0}^{\sharp} n_{0}^{-1}-i \vartheta_{1}\left(q_{0}^{b} / n_{0}\right)+\vartheta_{2}\left(r_{0} / n_{0}\right), \\
p_{k, 2}=-\frac{1}{k !} \psi V_{k}^{\sharp} n_{0}^{-(k+2) / 2}-\frac{i}{k !} \vartheta_{1}\left(q_{k}^{b} n_{0}^{-(k+2) / 2}\right)+\frac{1}{k !} \vartheta_{2}\left(\left(r_{k}-z n_{k}\right) n_{0}^{-(k+2) / 2}\right),
\end{gathered}
$$

for $j \geq 3$,

$$
p_{0, j}=-\vartheta_{j-2}\left(V_{0}^{\sharp} / n_{0}\right)-i \vartheta_{j-1}\left(q_{0}^{b} / n_{0}\right)+\vartheta_{j}\left(r_{0} / n_{0}\right),
$$

$$
p_{k, j}=-\frac{1}{k !} \vartheta_{j-2}\left(V_{k}^{\sharp} n_{0}^{-(k+2) / 2}\right)-\frac{i}{k !} \vartheta_{j-1}\left(q_{k}^{b} n_{0}^{-(k+2) / 2}\right)+\frac{1}{k !} \vartheta_{j}\left(\left(r_{k}-z n_{k}\right) n_{0}^{-(k+2) / 2}\right),
$$


for $k \geq 1, j \geq 3$. We will now transform the operator $\widetilde{P}_{\sharp}$ into the normal form studied in the previous section by using $h$-FIOs acting on the tangent variable $x^{\prime}$ only and independent of the normal variable $t$. Roughly speaking, we have to make a suitable symplectic change of the variables $\left(x^{\prime}, \xi^{\prime}\right)$ so that in the new coordinates our operator has a simpler form. Indeed, there exist an open, bounded domain $Y \subset \mathbf{R}^{d-1}$ and a symplectomorphism $\kappa: \mathcal{W}_{0} \rightarrow T^{*} Y$ such that if $(y, \eta):=\kappa\left(x^{\prime}, \xi^{\prime}\right)$, then

$$
\eta_{1}=\left(n_{0}^{-1} r_{0}\right)\left(x^{\prime}, \xi^{\prime}\right)-1
$$

and $\kappa\left(\Sigma \cap \mathcal{W}_{0}\right)=\left\{\eta_{1}=0\right\}$. Let $U=\mathcal{O}(1): L^{2}\left(\pi \mathcal{W}_{0}\right) \rightarrow L^{2}(Y)$ be an elliptic, zero-order $h-$ FIO associated to $\kappa$. Then the inverse $U^{-1}=\mathcal{O}(1): L^{2}(Y) \rightarrow L^{2}\left(\pi \mathcal{W}_{0}\right)$ is an $h$-FIO associated to the inverse symplectomorphism $\kappa^{-1}$. It is well-known (e.g. see Section 11 of [13]) that one can associate to $\kappa$ a linear map $\omega: C_{0}^{\infty}\left(\mathcal{W}_{0}\right) \rightarrow C_{0}^{\infty}\left(T^{*} Y\right)$ so that, if $a$ is a symbol independent of $h$, then

$$
U \mathrm{Op}_{h}(a) U^{-1}=\mathrm{Op}_{h}(\omega(a))+\mathcal{O}\left(h^{\infty}\right)
$$

where

$$
\omega(a) \sim \sum_{j=0}^{\infty} h^{j} \omega_{j}(a)
$$

with functions $\omega_{j}(a)$ independent of $h, \omega_{0}(a)=a \circ \kappa$. Moreover, each $\omega_{j}(a)$ is a linear combination of functions of the form $\left(\partial_{x^{\prime}}^{\alpha} \partial_{\xi^{\prime}}^{\beta} a\right) \circ \kappa$. Therefore $\omega_{j}(a)$ is supported in an arbitrary neighbourhood of the set $\kappa(\operatorname{supp} a)$. Observe now that

$$
\left(\psi n_{0}^{-1} r_{0}\right) \circ \kappa(y, \eta)=\left(\eta_{1}+1\right) \psi \circ \kappa(y, \eta)
$$

and $\psi \circ \kappa=1$ on $\operatorname{supp} \omega(\chi)$. Therefore, taking into account that $\mathcal{D}_{y_{1}}=\mathrm{Op}_{h}\left(\eta_{1}\right)$, we have modulo $\mathcal{O}\left(h^{\infty}\right)$

$$
\begin{gathered}
\mathrm{Op}_{h}(\chi)\left(\left(\psi n_{0}^{-1} r_{0}\right)\left(x^{\prime}, \mathcal{D}_{x^{\prime}}\right)-1\right) U^{-1} \\
=\mathrm{Op}_{h}(\chi) U^{-1}\left(\mathrm{Op}_{h}\left(\omega\left(\psi n_{0}^{-1} r_{0}\right)\right)-1\right) \\
=\mathrm{Op}_{h}(\chi) U^{-1} \mathrm{Op}_{h}\left(\omega\left(\psi n_{0}^{-1} r_{0}\right)-\omega_{0}\left(\psi n_{0}^{-1} r_{0}\right)\right) \\
+\mathrm{Op}_{h}(\chi) U^{-1}\left(\mathrm{Op}_{h}\left(\left(\eta_{1}+1\right) \psi \circ \kappa\right)-1\right) \\
=\mathrm{Op}_{h}(\chi) U^{-1} \mathrm{Op}_{h}\left(\omega\left(\psi n_{0}^{-1} r_{0}\right)-\omega_{0}\left(\psi n_{0}^{-1} r_{0}\right)\right) \\
+\mathrm{Op}_{h}(\chi) U^{-1}\left(\mathcal{D}_{y_{1}}+\mathrm{Op}_{h}\left(\left(\eta_{1}+1\right)(\psi \circ \kappa-1)\right)\right) \\
=\mathrm{Op}_{h}(\chi) U^{-1}\left(\mathcal{D}_{y_{1}}+\mathrm{Op}_{h}\left(\omega\left(\psi n_{0}^{-1} r_{0}\right)-\omega_{0}\left(\psi n_{0}^{-1} r_{0}\right)\right)\right) \\
+U^{-1} \mathrm{Op}_{h}(\omega(\chi)) \mathrm{Op}_{h}\left(\left(\eta_{1}+1\right)(\psi \circ \kappa-1)\right) \\
=\mathrm{Op}_{h}(\chi) U^{-1}\left(\mathcal{D}_{y_{1}}+\mathrm{Op}_{h}\left(\omega\left(\psi n_{0}^{-1} r_{0}\right)-\omega_{0}\left(\psi n_{0}^{-1} r_{0}\right)\right)\right) .
\end{gathered}
$$

Thus by (3.5) we get

$$
P_{\sharp} \mathrm{Op}_{h}(\chi) U^{-1}=\mathrm{Op}_{h}(\chi) U^{-1} P_{0}+\mathcal{O}\left(h^{\infty}\right)
$$

where $P_{0}$ is the operator from the previous section with $\mu=\operatorname{Im} z$ and

$$
m=\omega\left(p+\psi n_{0}^{-1} r_{0}\right)-\omega_{0}\left(\psi n_{0}^{-1} r_{0}\right) .
$$

It is easy to see that $m$ has the formal expansion

$$
m \sim \sum_{k=0}^{\infty} \sum_{j=0}^{\infty} t^{k} h^{j} m_{k, j}
$$


where $m_{0,0}=0$ and

$$
m_{0, j}=\omega_{j}\left(\psi n_{0}^{-1} r_{0}\right)+\sum_{\ell=0}^{j} \omega_{j-\ell}\left(p_{0, \ell}\right)
$$

for $j \geq 1$,

$$
m_{k, j}=\sum_{\ell=0}^{j} \omega_{j-\ell}\left(p_{k, \ell}\right)
$$

for $k \geq 1, j \geq 0$. In particular, we have

$$
m_{k, 0}=\frac{1}{k !}\left(\left(r_{k}-z n_{k}\right) n_{0}^{-(k+2) / 2} \psi\right) \circ \kappa, \quad k \geq 1 .
$$

Let $k \geq 0$ be an arbitrary integer and let $\widetilde{u}$ be the parametrix constructed in the previous section with

$$
\left.\widetilde{u}\right|_{t=0}=\mathrm{Op}_{h}\left(\phi \varrho^{k}\right) U \mathrm{Op}_{h}(\psi) f
$$

where $\phi=\phi\left(\eta_{1}\right)$ is the same function as in (2.3). Taking supp $\psi$ small enough we can arrange that $\phi=1$ on $\operatorname{supp} \omega(\psi)$. If $\widetilde{N}$ is the operator from the previous section, then

$$
\left.\mathcal{D}_{t} \widetilde{u}\right|_{t=0}=\tilde{N} \mathrm{Op}_{h}\left(\phi_{1} \varrho^{k}\right) U \mathrm{Op}_{h}(\psi) f
$$

where $\phi_{1}=\phi_{1}\left(\eta_{1}\right) \in C_{0}^{\infty}$ is such that $\phi_{1}=1$ on $\operatorname{supp} \phi$. Set

$$
v=\mathrm{Op}_{h}(\chi) U^{-1} \widetilde{u}
$$

We have

$$
\left.v\right|_{t=0}=\mathrm{Op}_{h}(\chi) U^{-1} \mathrm{Op}_{h}\left(\phi \varrho^{k}\right) U \mathrm{Op}_{h}(\psi) f
$$

and

$$
\left.\mathcal{D}_{t} v\right|_{t=0}=\mathrm{Op}_{h}(\chi) U^{-1} \tilde{N} \mathrm{Op}_{h}\left(\phi_{1} \varrho^{k}\right) U \mathrm{Op}_{h}(\psi) f .
$$

If we set $w=e^{\varphi^{\sharp}} v$, then

$$
\left.w\right|_{\partial X}=\mathrm{Op}_{h}(\chi) U^{-1} \mathrm{Op}_{h}\left(\phi \varrho^{k}\right) U \mathrm{Op}_{h}(\psi) f
$$

and, in view of (3),

$$
\left.\mathcal{D}_{\nu} w\right|_{\partial X}=\mathcal{T}^{(k)} f
$$

where

$$
\begin{aligned}
& \mathcal{T}^{(k)}=n_{0}^{1 / 2} \mathrm{Op}_{h}(\chi) U^{-1} \tilde{N} \mathrm{Op}_{h}\left(\phi_{1} \varrho^{k}\right) U \mathrm{Op}_{h}(\psi)+\frac{i h}{2} q^{\sharp}\left(0, x^{\prime}\right) \mathrm{Op}_{h}(\chi) U^{-1} \mathrm{Op}_{h}\left(\phi \varrho^{k}\right) U \mathrm{Op}_{h}(\psi) \\
& =\sum_{j=0}^{M+1} h^{j} \mathcal{T}_{j}^{(k)}
\end{aligned}
$$

where

$$
\begin{gathered}
\mathcal{T}_{0}^{(k)}=n_{0}^{1 / 2} \mathrm{Op}_{h}(\chi) U^{-1} \mathrm{Op}_{h}\left(\phi \varrho^{k+1}\right) U \mathrm{Op}_{h}(\psi), \\
\mathcal{T}_{1}^{(k)}=\frac{i}{2} q^{\sharp}\left(0, x^{\prime}\right) \mathrm{Op}_{h}(\chi) U^{-1} \mathrm{Op}_{h}\left(\phi \varrho^{k}\right) U \mathrm{Op}_{h}(\psi)-i n_{0}^{1 / 2} \mathrm{Op}_{h}(\chi) U^{-1} \mathrm{Op}_{h}\left(\varrho^{k} a_{1,0}\right) U \mathrm{Op}_{h}(\psi), \\
\mathcal{T}_{j}^{(k)}=-i n_{0}^{1 / 2} \mathrm{Op}_{h}(\chi) U^{-1} \mathrm{Op}_{h}\left(\varrho^{k} a_{1, j-1}\right) U \mathrm{Op}_{h}(\psi),
\end{gathered}
$$

for $j \geq 2$. Set

$$
\begin{gathered}
\mathcal{H}_{j}^{(k)}=n_{0}^{1 / 2} \mathrm{Op}_{h}(\chi) U^{-1} \mathrm{Op}_{h}\left(\varrho^{k-j-1}\left(n_{j} n_{0}^{-(j+2) / 2} \psi\right) \circ \kappa\right) U \mathrm{Op}_{h}(\psi), \\
\mathcal{K}_{j}^{(k)}=n_{j} n_{0}^{-(j+1) / 2} \operatorname{Op}_{h}(\chi) U^{-1} \mathrm{Op}_{h}\left(\phi \varrho^{k-j-1}\right) U \mathrm{Op}_{h}(\psi)
\end{gathered}
$$


Lemma 3.2. For $0 \leq k \leq j+1$ we have the bound

$$
\left\|\mathcal{H}_{j}^{(k)}-\mathcal{K}_{j}^{(k)}\right\| \lesssim h|\operatorname{Im} z|^{-(j+1-k) / 2} .
$$

Proof. Clearly

$$
\varrho^{k-j-1}\left(n_{j} n_{0}^{-(j+2) / 2} \psi\right) \circ \kappa, \phi \varrho^{k-j-1} \in S^{-(j+1-k)}(|\varrho|) \subset S^{-(j+1-k) / 2}(|\mu|)
$$

and hence

$$
\mathrm{Op}_{h}\left(\varrho^{k-j-1}\left(n_{j} n_{0}^{-(j+2) / 2} \psi\right) \circ \kappa\right), \mathrm{Op}_{h}\left(\phi \varrho^{k-j-1}\right)=\mathcal{O}\left(|\operatorname{Im} z|^{-(j+1-k) / 2}\right)
$$

in the $L^{2}(Y) \rightarrow L^{2}(Y)$ norm. On the other hand, we have, $\bmod \mathcal{O}(h)$,

$$
\begin{aligned}
& n_{j} n_{0}^{-(j+1) / 2} \mathrm{Op}_{h}(\chi) U^{-1}=n_{0}^{1 / 2} \mathrm{Op}_{h}(\chi) \mathrm{Op}_{h}\left(n_{j} n_{0}^{-(j+2) / 2} \psi\right) U^{-1} \\
& =n_{0}^{1 / 2} \mathrm{Op}_{h}(\chi) U^{-1}\left(\mathrm{Op}_{h}\left(\left(n_{j} n_{0}^{-(j+2) / 2} \psi\right) \circ \kappa\right)+\mathcal{O}(h)\right) .
\end{aligned}
$$

Therefore, modulo an operator of norm $\mathcal{O}\left(h|\operatorname{Im} z|^{-(j+1-k) / 2}\right)$, we obtain

$$
\begin{gathered}
\mathcal{H}_{j}^{(k)}-\mathcal{K}_{j}^{(k)}=n_{0}^{1 / 2} \mathrm{Op}_{h}(\chi) U^{-1}\left(\operatorname{Op}_{h}\left(\varrho^{k-j-1}\left(n_{j} n_{0}^{-(j+2) / 2} \psi\right) \circ \kappa\right)\right. \\
\left.-\mathrm{Op}_{h}\left(\left(n_{j} n_{0}^{-(j+2) / 2} \psi\right) \circ \kappa\right) \mathrm{Op}_{h}\left(\phi \varrho^{k-j-1}\right)\right) U \mathrm{Op}_{h}(\psi) .
\end{gathered}
$$

Since $\phi=1$ on $\operatorname{supp} \psi \circ \kappa$, it is easy to see that the bound (3.10) follows from Proposition 2.1.

Lemma 3.3. For every $j \geq 1$ the operator $\mathcal{T}_{j}^{(k)}+i(-2 i)^{-j-1} z \mathcal{H}_{j}^{(k)}$ is independent of all $n_{\ell}$ with $\ell \geq j$.

Proof. By Lemma 2.6 the function

$$
a_{1, j-1}+\frac{j ! \phi m_{j, 0}}{(-2 i \varrho)^{j+1}}
$$

depends only on $m_{\nu, 0}$ with $\nu \leq j-1$ and $m_{\nu, \ell}$ with $1 \leq \ell \leq j, \nu \leq j-\ell \leq j-1$. On the other hand, it is clear from the above formulas that $m_{\nu, \ell}$ with $\nu \leq j-1$ depends only on $n_{\ell}$ with $\ell \leq j-1$. Thus by (3.7) we conclude that

$$
a_{1, j-1}-z(-2 i \varrho)^{-j-1}\left(n_{j} n_{0}^{-(j+2) / 2} \psi\right) \circ \kappa
$$

is independent of all $n_{\ell}$ with $\ell \geq j$, which clearly implies the lemma.

Proposition 2.8 and Lemma 2.9 imply the following

Proposition 3.4. Given any integers $s, k \geq 0$ such that $k \leq 3 s+2$, we have the estimate

$$
\left\|N(h, \mu) \mathrm{Op}_{h}(\chi) U^{-1} \mathrm{Op}_{h}\left(\phi \varrho^{k}\right) U \mathrm{Op}_{h}(\psi)-\sum_{j=0}^{s} h^{j} \mathcal{T}_{j}^{(k)}\right\| \lesssim h^{s+1}|\operatorname{Im} z|^{-(3 s+2-k) / 2} .
$$

For $j \geq 1,0 \leq k \leq 3 j-1$ we also have

$$
\left\|\mathcal{T}_{j}^{(k)}\right\| \lesssim|\operatorname{Im} z|^{-(3 j-1-k) / 2}
$$


Proof. Clearly (3.12) follows from (2.26) used with $j$ replaced by $j-1$. To prove (3.11) we will make use of the coercivity of the Dirichlet realization, $G_{D}$, of the operator $n^{-1} \Delta_{X}$ in the Hilbert space $L^{2}(X ; n d x)$. We have

$$
\|u\|_{H_{h}^{2}(X)} \lesssim\left\|h^{2} G_{D} u\right\|_{L^{2}(X)}+\|u\|_{L^{2}(X)}, \quad \forall u \in D\left(G_{D}\right),
$$

where $H_{h}^{2}(X)$ denotes the Sobolev space equipped with the semi-classical norm. This together with the semi-classical version of the trace theorem imply

$$
\begin{gathered}
\left\|\gamma \mathcal{D}_{\nu}\left(h^{2} G_{D}+z\right)^{-1}\right\|_{L^{2}(X) \rightarrow L^{2}(\partial X)} \lesssim h^{-1 / 2}\left\|\left(h^{2} G_{D}+z\right)^{-1}\right\|_{L^{2}(X) \rightarrow H_{h}^{2}(X)} \\
\lesssim h^{-1 / 2}+h^{-1 / 2}\left\|\left(h^{2} G_{D}+z\right)^{-1}\right\|_{L^{2}(X) \rightarrow L^{2}(X)} \lesssim h^{-1 / 2}|\operatorname{Im} z|^{-1} \lesssim h^{-7 / 6}
\end{gathered}
$$

where $\gamma$ denotes the restriction on $\partial X$. Let $u$ be the solution to equation (1.2) with boundary condition

$$
\left.u\right|_{\partial X}=\mathrm{Op}_{h}(\chi) U^{-1} \mathrm{Op}_{h}\left(\phi \varrho^{k}\right) U \mathrm{Op}_{h}(\psi) f .
$$

If $v, w$ and $\widetilde{u}$ are the functions introduced above, we have in view of (3.6) and (3.8), that $\left.(u-w)\right|_{\partial X}=0$ and

$$
\left(h^{2} n^{-1} \Delta_{X}+z\right)(u-w)=P(h) w=P_{\sharp}(h) v=\mathrm{Op}_{h}(\chi) U^{-1} P_{0} \widetilde{u}+\mathcal{O}\left(h^{\infty}\right) \widetilde{u} .
$$

Hence

$$
u-w=\left(h^{2} G_{D}+z\right)^{-1}\left(\operatorname{Op}_{h}(\chi) U^{-1} P_{0}+\mathcal{O}\left(h^{\infty}\right)\right) \widetilde{u}
$$

which together with (3.9) imply the identity

$$
\begin{gathered}
N \operatorname{Np}_{h}(\chi) U^{-1} \operatorname{Op}_{h}\left(\phi \varrho^{k}\right) U \mathrm{Op}_{h}(\psi) f-\mathcal{T}^{(k)} f \\
=\gamma \mathcal{D}_{\nu}\left(h^{2} G_{D}+z\right)^{-1}\left(\operatorname{Op}_{h}(\chi) U^{-1} P_{0}+\mathcal{O}\left(h^{\infty}\right)\right) \widetilde{u} .
\end{gathered}
$$

By Proposition 2.8, (3) and (3) we get

$$
\begin{gathered}
\left\|N \operatorname{NOp}_{h}(\chi) U^{-1} \operatorname{Op}_{h}\left(\phi \varrho^{k}\right) U \mathrm{Op}_{h}(\psi) f-\mathcal{T}^{(k)} f\right\|_{L^{2}(\partial X)} \\
\lesssim h^{-7 / 6}\left\|P_{0} \widetilde{u}\right\|_{L^{2}((0,1) \times Y)}+\mathcal{O}\left(h^{\infty}\right)\|\widetilde{u}\|_{L^{2}((0,1) \times Y)} \\
\lesssim h^{\epsilon M / 2-7 / 6}\left\|\mathrm{Op}_{h}\left(\phi \varrho^{k}\right) U \mathrm{Op}_{h}(\psi) f\right\|_{L^{2}(Y)} \lesssim h^{\epsilon M / 2-7 / 6}\|f\|_{L^{2}(\partial X)} .
\end{gathered}
$$

On the other hand, by Lemma 2.9 we have

$$
\left\|\mathcal{T}^{(k)}-\sum_{j=0}^{s} h^{j} \mathcal{T}_{j}^{(k)}\right\| \lesssim h^{s+1}|\operatorname{Im} z|^{-(3 s+2-k) / 2} .
$$

Take now $M$ so that $\epsilon M / 2-7 / 6>s+1$. Clearly, the bound (3.11) follows from (3.15) and (3.16).

It follows from Lemma 3.3 that for $s \geq 1$ the operator

$$
\mathcal{P}_{s}^{(k)}=\sum_{j=0}^{s} h^{j} \mathcal{T}_{j}^{(k)}+i(-2 i)^{-s-1} z h^{s} \mathcal{H}_{s}^{(k)}
$$


is independent of all $n_{\ell}$ with $\ell \geq s$. Put $\mathcal{P}_{0}^{(0)}=\mathcal{T}_{0}^{(0)}$. By (3.10) and (3.11) we have for $s=k=0$ and $s \geq 1,0 \leq k \leq s+1$,

$$
\left\|N \mathrm{~Np}_{h}(\chi) U^{-1} \mathrm{Op}_{h}\left(\phi \varrho^{k}\right) U \mathrm{Op}_{h}(\psi)-\mathcal{P}_{s}^{(k)}-z c_{s} h^{s} \mathcal{K}_{s}^{(k)}\right\| \lesssim h^{s+1}|\operatorname{Im} z|^{-(3 s+2-k) / 2}
$$

where $c_{s}$ is as in Theorem 3.1. Since

$$
\mathrm{Op}_{h}(\chi) U^{-1} \mathrm{Op}_{h}(\phi) U \mathrm{Op}_{h}(\psi)=\mathrm{Op}_{h}(\chi)+\mathcal{O}\left(h^{\infty}\right),
$$

it is easy to see that (3.1) follows from (3.17) with $k=0$ and $\mathcal{A}_{s}=\mathcal{P}_{s}^{(0)}-\mathcal{T}_{0}^{(0)}$. Since

$$
\begin{gathered}
n_{s} n_{0}^{-(s+1) / 2} \mathrm{Op}_{h}(\chi) U^{-1} \mathrm{Op}_{h}(\phi) U \mathrm{Op}_{h}(\psi) \\
=n_{0}^{-(s+1) / 2} \mathrm{Op}_{h}\left(n_{s} \chi\right)+\mathcal{O}(h)=\mathrm{Op}_{h}\left(n_{s} \chi\right) n_{0}^{-(s+1) / 2}+\mathcal{O}(h),
\end{gathered}
$$

the bound (3.2) follows from (3.17) with $k=s+1$ and

$$
\begin{gathered}
\mathcal{B}_{s}^{R}=\left(z c_{s} h^{s}\right)^{-1} \operatorname{Op}_{h}(\chi) U^{-1} \operatorname{Op}_{h}\left(\phi \varrho^{s+1}\right) U \mathrm{Op}_{h}(\psi) n_{0}^{(s+1) / 2}, \\
\mathcal{C}_{s}^{R}=\left(z c_{s} h^{s}\right)^{-1} \mathcal{P}_{s}^{(s+1)} n_{0}^{(s+1) / 2}
\end{gathered}
$$

Since

$$
N(h, z)=N(h, \bar{z})^{*}
$$

and

$$
\mathrm{Op}_{h}\left(n_{s} \chi\right)=\left(\mathrm{Op}_{h}\left(n_{s} \chi\right)\right)^{*}+\mathcal{O}(h),
$$

the bound (3.3) follows from (3.2) with

$$
\mathcal{B}_{s}^{L}(h, z)=\mathcal{B}_{s}^{R}(h, \bar{z})^{*}, \quad \mathcal{C}_{s}^{L}(h, z)=\mathcal{C}_{s}^{R}(h, \bar{z})^{*} .
$$

The analysis of the operator $\mathrm{NOp}_{h}(1-\chi)$ is much easier and, as mentioned in the introduction, can be done for $|\operatorname{Im} z| \geq h^{1-\epsilon}$. Indeed, in this case the parametrix construction in Section 3 of 12. gives full expansions similar to those in Theorem 3.1 but with better bounds in the righthand sides (with $|\operatorname{Im} z|$ replaced by 1). Thus, combining the results of [12] with Theorem 3.1 we get the following

Theorem 3.5. Let $|\operatorname{Im} z| \geq h^{2 / 3-\epsilon}, 0<\epsilon \ll 1$. Then, for every integer $s \geq 0$ there is an operator

$$
\mathcal{A}_{s}(h, z)=\mathcal{O}\left(h|\operatorname{Im} z|^{-1}\right): L^{2}(\partial X) \rightarrow L^{2}(\partial X)
$$

independent of all $n_{\ell}$ with $\ell \geq s$ such that

$$
\left\|N(h, z)-\widetilde{\mathrm{Op}}_{h}\left(\rho+c_{s} h^{s} \rho^{-s-1} z n_{s}\right)-\mathcal{A}_{s}\right\| \lesssim h^{s+1}|\operatorname{Im} z|^{-(3 s+2) / 2}
$$

where $c_{s}=0, \mathcal{A}_{s}=0$ if $s=0$, and $c_{s}=-i(-2 i)^{-s-1}$ for $s \geq 1$. Furthermore, for every integer $s \geq 1$ there are operators $\mathcal{B}_{s}^{R}(h, z), \mathcal{B}_{s}^{L}(h, z)$ independent of all $n_{\ell}$ with $\ell \geq 1$, and operators $\mathcal{C}_{s}^{R}(h, z), \mathcal{C}_{s}^{L}(h, z)$ independent of all $n_{\ell}$ with $\ell \geq s$ such that

$$
\begin{gathered}
\left\|N(h, z) \mathcal{B}_{s}^{R}-\mathcal{C}_{s}^{R}-n_{s} I\right\| \lesssim h|\operatorname{Im} z|^{-(2 s+1) / 2}, \\
\left\|\mathcal{B}_{s}^{L} N(h, z)-\mathcal{C}_{s}^{L}-n_{s} I\right\| \lesssim h|\operatorname{Im} z|^{-(2 s+1) / 2},
\end{gathered}
$$

where I denotes the identity. 


\section{Applications to the transmission eigenvalues}

Let $\Omega \subset \mathbf{R}^{d}, d \geq 2$, be a bounded, connected domain with a $C^{\infty}$ smooth boundary $\Gamma=\partial \Omega$. A complex number $\lambda \neq 0, \operatorname{Re} \lambda \geq 0$, will be said to be a transmission eigenvalue if the following problem has a non-trivial solution:

$$
\left\{\begin{array}{cc}
\left(\Delta+\lambda^{2} n_{1}(x)\right) u_{1}=0 & \text { in } \quad \Omega, \\
\left(\Delta+\lambda^{2} n_{2}(x)\right) u_{2}=0 & \text { in } \quad \Omega, \\
u_{1}=u_{2}, \partial_{\nu} u_{1}=\partial_{\nu} u_{2} & \text { on } \quad \Gamma,
\end{array}\right.
$$

where $\nu$ denotes the Euclidean unit inner normal to $\Gamma, n_{j} \in C^{\infty}(\bar{\Omega}), j=1,2$ are strictly positive real-valued functions. We have the following

Theorem 4.1. Suppose that there is an integer $j \geq 1$ such that

$$
\begin{gathered}
\partial_{\nu}^{s} n_{1}(x) \equiv \partial_{\nu}^{s} n_{2}(x) \quad \text { on } \quad \Gamma, \quad 0 \leq s \leq j-1, \\
\partial_{\nu}^{j} n_{1}(x) \neq \partial_{\nu}^{j} n_{2}(x) \quad \text { on } \Gamma .
\end{gathered}
$$

Then there exists a constant $C>0$ such that there are no transmission eigenvalues in the region

$$
\left\{\lambda \in \mathbf{C}: \operatorname{Re} \lambda \geq 0,|\operatorname{Im} \lambda| \geq C(\operatorname{Re} \lambda+1)^{1-k_{j}}\right\},
$$

where $k_{1}=2 / 3-\epsilon, \forall 0<\epsilon \ll 1$, and $k_{j}=2(2 j+1)^{-1}$ if $j \geq 2$.

Note that this theorem has been proved in [12] with $k_{j}=2(3 j+2)^{-1}, j \geq 1$. Here we get a larger eigenvalue-free region. Previously, smaller eigenvalue-free regions were obtained in [4]. Theorem 4.1 is an immediate consequence of the estimate (3.20) above. The proof goes in precisely the same way as in Section 5 of [12] using (3.20) instead of Theorem 4.1 of [12], and therefore we omit the details here.

In the non-degenerate isotropic case when

$$
n_{1}(x) \neq n_{2}(x) \quad \text { on } \quad \Gamma
$$

it has been proved in [11] that there are no transmission eigenvalues in a much larger region of the form

$$
\{\lambda \in \mathbf{C}: \operatorname{Re} \lambda \geq 0,|\operatorname{Im} \lambda| \geq C\}
$$

for some constant $C>0$, which is in fact the optimal eigenvalue-free region. Note that it follows from 1 (see Theorem 4.2) that under the condition (4.2) the eigenvalue-free region (4.6) is no longer valid. Note also that parabolic eigenvalue-free regions imply Weyl asymptotics for the counting function of the transmission eigenvalues with remainder term depending on the size of the eigenvalue-free region (see [7]). Roughly, the larger the eigenvalue-free region is, the smaller the remainder term is.

\section{REFERENCES}

[1] D. Colton, Y.-J. Leung And S. Meng, Distribution of complex transmission eigenvalues for spherically stratified media, Inverse problems 31 (2015), 035006.

[2] M. Dimassi And J. Suöstrand, Spectral asymptotics in semi-classical limit, London Mathematical Society, Lecture Notes Series, 268, Cambridge University Press, 1999.

[3] J. Galkowski, The quantum Sabine law for resonances in transmission problems, preprint 2016.

[4] E. Lakshtanov and B. Vainberg, Application of elliptic theory to the isotropic interior transmission eigenvalue problem, Inverse Problems 29 (2013), 104003. 
[5] J. SJöstrand, Weyl law for semi-classical resonances with randomly perturbed potentials, Mémoire de la Societé Mathématique de France, vol. 136 (2014).

[6] J. Sjöstrand And M. Zworski, Asymptotic distribution of resonances for convex obstacles, Acta Math. 183 (1999), 191-253.

[7] V. Petkov and G. Vodev, Asymptotics of the number of the interior transmission eigenvalues, J. Spectral Theory 7(1) (2017), 1-31.

[8] G. Popov And G. Vodev, Resonances near the real axis for transpaent obstacles, Comm. Math. Phys. 207 (1999), 411-438.

[9] G. Vodev, Transmission eigenvalue-free regions, Comm. Math. Phys. 336 (2015), 1141-1166.

[10] G. Vodev, Transmission eigenvalues for strictly concave domains, Math. Ann. 366 (2016), 301-336.

[11] G. VodEv, High-frequency approximation of the interior Dirichlet-to-Neumann map and applications to the transmission eigenvalues, Anal. PDE 11(1) (2018), 213-236.

[12] G. Vodev, Parabolic transmission eigenvalue-free regions in the degenerate isotropic case, Asympt. Anal. 106 (2018), 147-168.

[13] M. Zworski, Semi-classical Analysis, American Mathematical Society, Providence, RI, 2012.

Université de Nantes, Laboratoire de Mathématiques Jean Leray, 2 rue de la Houssinière, BP 92208, 44322 Nantes Cedex 03, France

E-mail address: Georgi.Vodev@univ-nantes.fr 